\title{
Mechanistic insight into substrate processing and allosteric inhibition of human p97
}

\author{
Man Pan ${ }^{1,3 \bowtie}$, Yuanyuan $\mathbf{Y u}^{1,3}$, Huasong $\mathrm{Ai}^{2}$, Qingyun Zheng ${ }^{2}$, Yuan Xie ${ }^{1}{ }^{1}$, Lei Liu ${ }^{2} \bowtie$ and

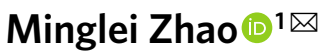

\begin{abstract}
p97 processes ubiquitinated substrates and plays a central role in cellular protein homeostasis. Here, we report a series of cryo-EM structures of the substrate-engaged human p97 complex with resolutions ranging from 2.9 to $3.8 \AA$ that captured 'power-stroke'-like motions of both the D1 and D2 ATPase rings of p97. A key feature of these structures is the critical conformational changes of the intersubunit signaling (ISS) motifs, which tighten the binding of nucleotides and neighboring subunits and contribute to the spiral staircase conformation of the D1 and D2 rings. In addition, we determined the cryo-EM structure of human $p 97$ in complex with NMS-873, a potent p97 inhibitor, at a resolution of $2.4 \AA$. The structures showed that NMS-873 binds at a cryptic groove in the D2 domain and interacts with the ISS motif, preventing its conformational change and thus blocking substrate translocation allosterically.
\end{abstract}

$p^{s}$ 97 is a $540-\mathrm{kDa}$ homohexameric protein that belongs to the family of ATPases associated with diverse cellular activities (AAA) ${ }^{1-3}$. It undergoes repetitive ATP hydrolysis and conformational changes to extract ubiquitinated substrates from various cellular components, such as membranes, ribosomes and chromatin ${ }^{4}$. p97 plays a central role in many cellular processes, including endoplasmic reticulum-associated degradation, mitochondrial-associated degradation, chromatin-associated degradation, autophagy and endosomal trafficking ${ }^{5}$. Mutations in human p97 have been found in multisystem proteinopathy (MSP), a dominantly inherited degenerative disorder that can affect muscle, bone and the central nervous system, and which can manifest clinically as inclusion body myopathy with early-onset Paget disease and frontotemporal dementia (IBMPFD) and familial amyotrophic lateral sclerosis (ALS) ${ }^{6,7}$. Moreover, recent studies have also targeted p97 for the treatment of cancer and viral infections $s^{8,9}$.

p97 has two tandem AAA domains named D1 and D2 and an additional $\mathrm{N}$ domain at the $\mathrm{N}$ terminus (Fig. 1a). This protein functions as a homomeric hexamer, with 12 copies of ATPase domains organized into two rings ${ }^{10}$. Crystal structures of full-length $\mathrm{p} 97$ were determined more than a decade ago ${ }^{11,12}$. High-resolution single-particle cryo-EM structures have also been reported ${ }^{13,14}$. However, all the structures of $\mathrm{p} 97$ determined thus far adopt a six-fold symmetric conformation, providing limited information regarding the mechanism, since almost all AAA proteins, including close homologs of p97, break symmetry when engaged with substrates $^{15-17}$. Recent cryo-EM studies of Cdc48, the yeast homolog of p97, in complex with either in vivo ${ }^{18}$ or in vitro ${ }^{19}$ substrates provided crucial insight into the working states. However, with medium resolution ( $3.7 \AA$ to $4.2 \AA$ ) and limited conformational states, critical mechanistic questions, such as how the AAA domains in two rings coordinate to achieve translocation, remain to be answered. In addition, owing to its biomedical importance, many small-molecule inhibitors of p97 have been developed in the past decade ${ }^{8,20,21}$. NMS873, a noncompetitive allosteric inhibitor of p97 (ref. ${ }^{21}$ ), has been shown to be active in tumor cell lines and fibroblasts of patients with IBMPFD ${ }^{21,22}$. More importantly, low nanomolar concentrations of NMS-873 were recently reported to inhibit the replication of several viruses, including severe acute respiratory syndrome coronavirus 2 (SARS-CoV-2) and influenza virus, through its inhibition of p97 (refs. ${ }^{23-25}$ ). Little is known regarding the allosteric inhibition mechanism due to the lack of a substrate-engaged human $\mathrm{p} 97$ structure in a working state, which is of considerable biological and clinical interest.

In this study, starting from a fully assembled human p97 complex with the cofactor Npl4/Ufd 1 and a substrate, we triggered ATP hydrolysis and determined a series of substrate-engaged p97 structures with resolutions ranging from 2.9 to $3.8 \AA$ using single-particle cryo-EM. The structures captured 'power-stroke'-like motions of both the D1 and D2 rings of p97 driven by ATP hydrolysis, and elucidated how the two rings coordinated to achieve translocation through ISS motifs, the pore loops and N- and C-terminal tails. We further determined the cryo-EM structure of $\mathrm{p} 97$ in the presence of NMS-873 at a resolution of $2.4 \AA$ and uncovered a novel inhibition mechanism. Finally, using NMS-873 at a substoichiometric concentration, we captured a series of intermediate states, suggesting how p97 coordinates with its cofactor Npl4 to initiate substrate translocation. Taken together, our results establish a comprehensive understanding of substrate processing and allosteric inhibition of human p97.

\section{Results}

Working states of human p97. First, we assembled complexes of p97 from recombinantly overexpressed components without introducing additional ATP. Two mutations, A232E and E578Q, were introduced into the human p97 construct to stabilize the complex, as previously described ${ }^{26}$. The A232E mutation has been found in patients with MSP and has been reported as an activating mutant with its $\mathrm{N}$ domains shifted toward the 'up conformation ${ }^{27}$. E578Q is a mutation in the Walker B motif of the D2 domain that slows substrate unfolding ${ }^{19,28}$. Combined, these two mutations decreased but maintained the unfolding activity of p97 (Extended Data Fig. 1a)

'Department of Biochemistry and Molecular Biology, The University of Chicago, Chicago, IL, USA. ${ }^{2}$ Tsinghua-Peking Center for Life Sciences, Department of Chemistry, Tsinghua University, Beijing, China. ${ }^{3}$ These authors contributed equally: Man Pan, Yuanyuan Yu. ${ }_{\text {e-mail: panm@uchicago.edu; }}$

Iliu@mail.tsinghua.edu.cn; mlzhao@uchicago.edu 
a

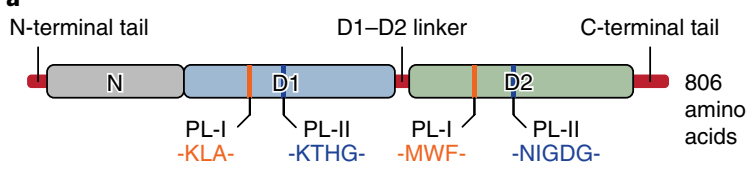

b

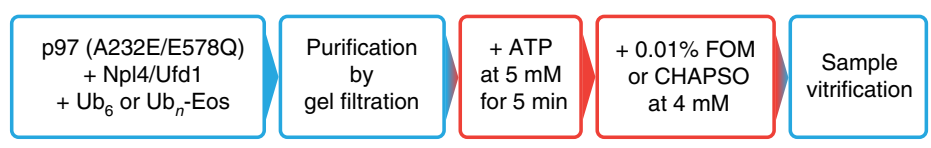

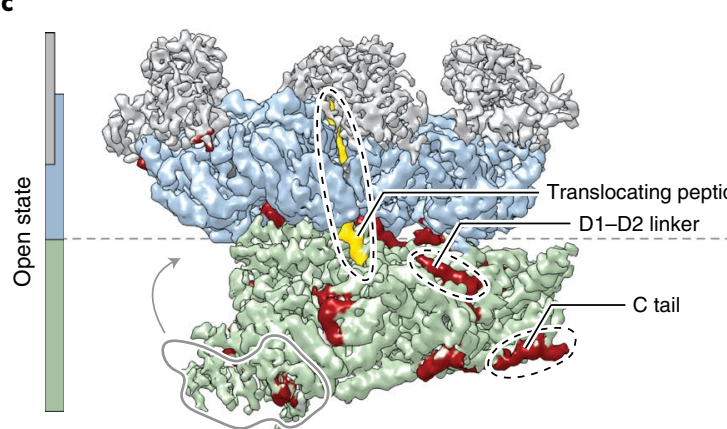
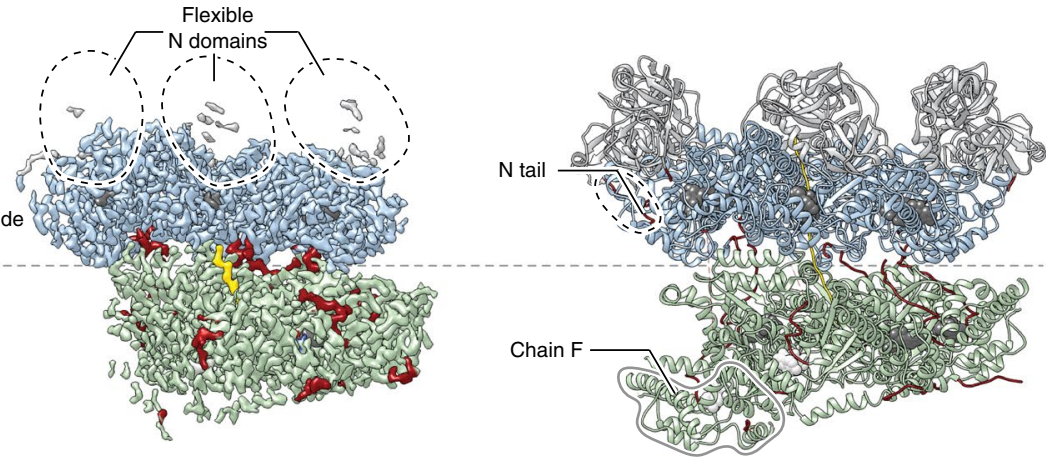

d

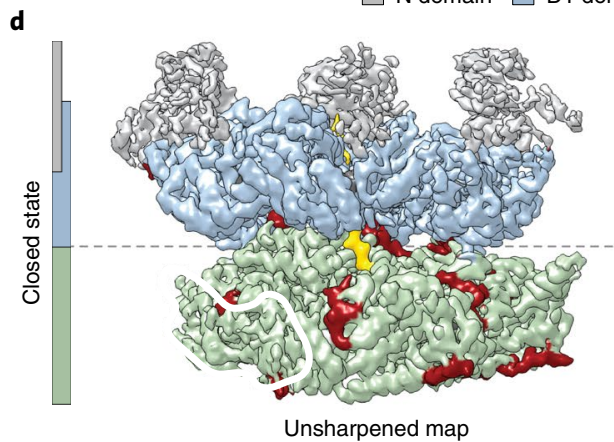

D2 domain

Tail and linker $\square$ Translocating peptide

\begin{abstract}
$\square$ ATP $\square$ ADP
\end{abstract}

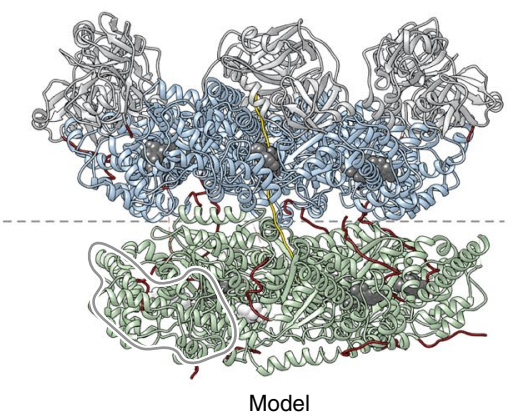

e

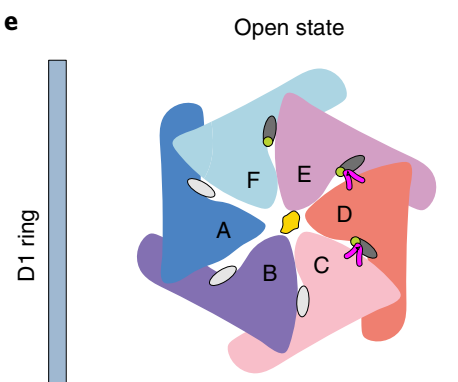

Closed state
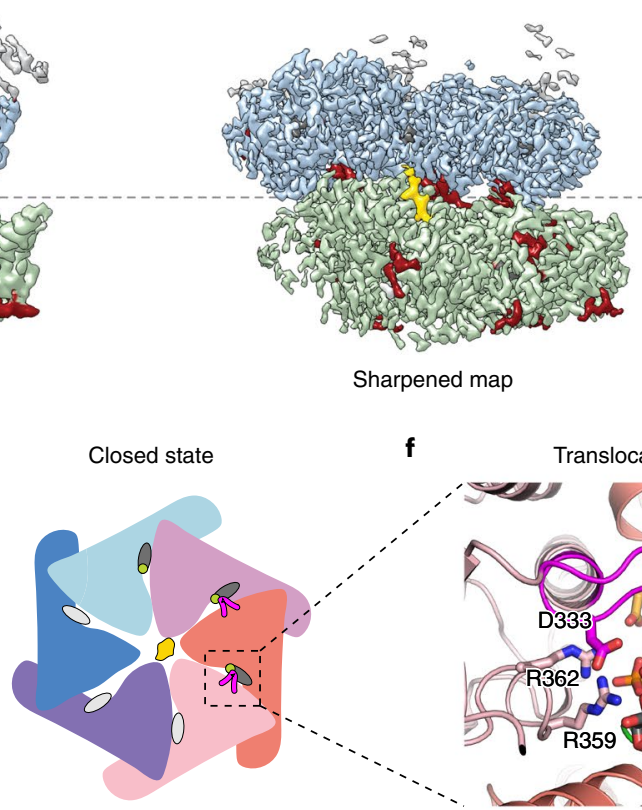

Translocating
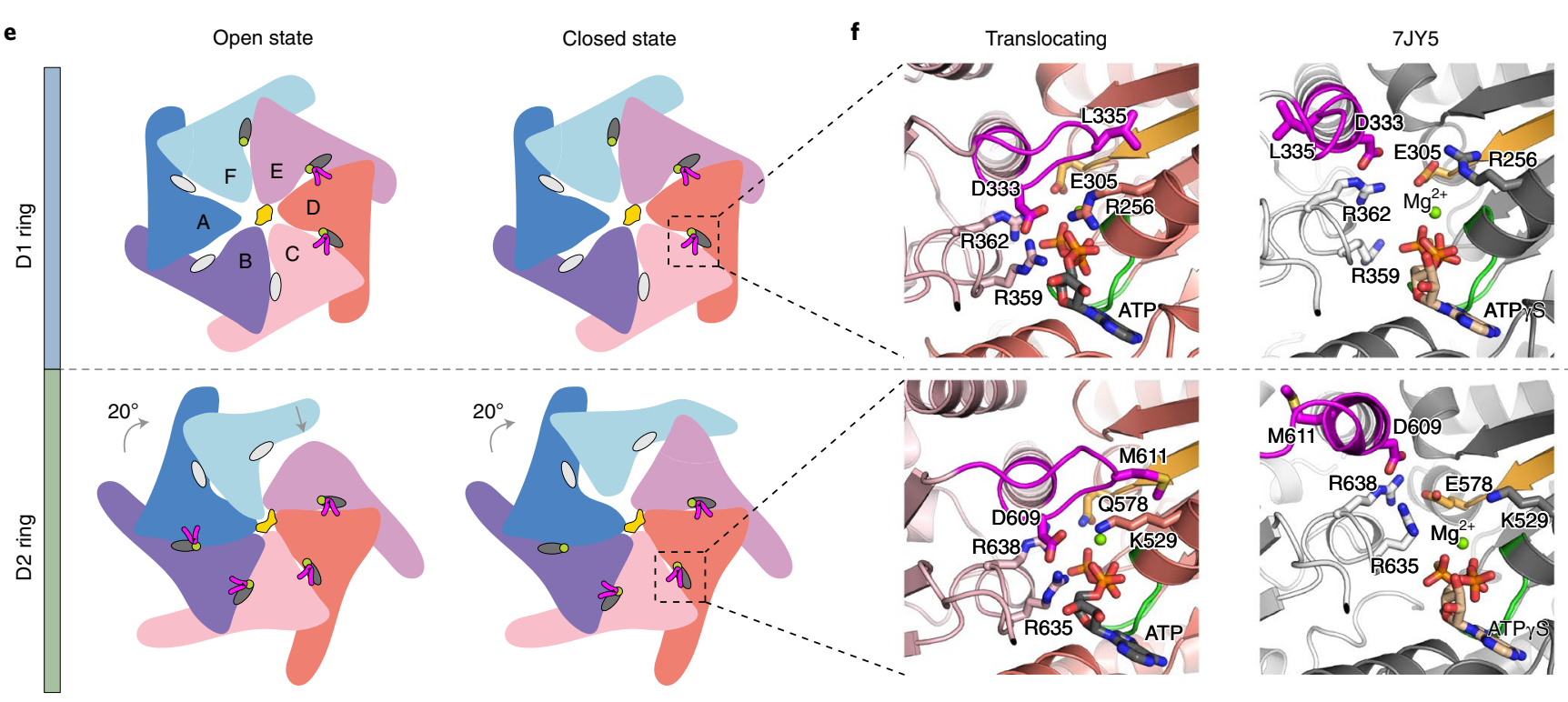

Translocating peptide $\square$ ISS motif $\square$ ATP $\square$ ADP $\square \mathrm{Mg}^{2+}$

$\square$ Walker A motif $\square$ Walker B motif

$\square$ ATP $\gamma \mathrm{S}$

ISS motif

Fig. 1 | Structures of human $\mathbf{p 9 7}$ in working states. a, Domain architecture of human $\mathrm{p} 97$. This color code for individual domains is used throughout the article whenever possible. Pore loop residues in D1 and D2 domains are listed. b. Sample preparation procedures. Steps performed at $4{ }^{\circ} \mathrm{C}$ are in blue boxes. Steps performed at room temperature are in red boxes. c,d, Cryo-EM maps and models of human p97 engaged with a translocating peptide. The maps and models are aligned. Contour level: unsharpened map, 0.015; sharpened map, 0.035. Open state (c) and closed state (d). e, Illustrations showing the arrangement of the D1 and D2 rings in the open and closed states, respectively. The ATPase rings were projected along the central pore. $\mathbf{f}$, Magnified views of compressed ATP-binding sites in the D1 and D2 rings. The corresponding ATP $\gamma$ S-binding sites from the nontranslocating structure (PDB 7JY5) are shown for comparison.

and minimized the conformational heterogeneity resulting from the $\mathrm{N}$ domains. All the structural work in this study used the p97 (A232E/E578Q) mutant and Npl4/Ufd1 as the cofactors.
For substrates, either polyubiquitinated $\mathrm{Ub}$-Eos $\left(\mathrm{Ub}_{n}\right.$-Eos) or K48-linked hexa-ubiquitin $\left(\mathrm{Ub}_{6}\right)$ was used. The assembled complex, named p97-Npl4/Ufd1-Ub - Eos or p97-Npl4/Ufd1-Ub , eluted as 


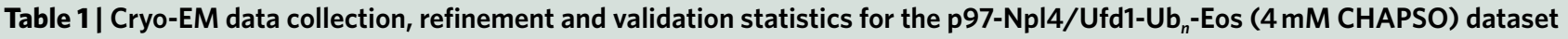

Class 1 (closed state)
(EMD-23449, PDB 7LN5)

$\times 81,000$
300
50
-1.0 to -2.5
1.063
$\mathrm{C} 1$

672,284

Class 2 (open state)

(EMD-23450, PDB 7LN6)
Class 3

(EMD-23451)
215,530

3.09

0.143

3.33

0.5

$-79.7$

35,051

4,447

Protein residues

$\mathrm{Mg}, 7 ; \mathrm{ADP}, 5 ;$ ATP, 7

$0.48 / 75.21 / 37.58$

$11.67 / 65.09 / 33.22$

0.003

0.617

Ligands

$B$ factors $\left(\AA^{2}\right)$

Protein

Ligand

R.m.s. deviations

Bond lengths $(\AA)$

41,239

3.58

0.143

3.85

0.5

$-79.7$

35,072

4,456

$\mathrm{Mg}, 7 ; \mathrm{ADP}, 5 ; \mathrm{ATP}, 7$

$0.36 / 49.31 / 19.03$

$2.26 / 41.30 / 13.85$

0.004

0.673

Validation

MolProbity score

Clashscore

1.52

9.95

0.03

Poor rotamers (\%)

Favored (\%)

98.46

Allowed (\%)

1.54

Disallowed (\%)

0.00
1.68

10.93

0.03

97.35

2.63

0.02
49,294

3.77

0.143 single peaks in the size-exclusion chromatograms (Extended Data Fig. 1b,c). To capture the working states of p97, ATP hydrolysis was triggered by adding $5 \mathrm{mM}$ ATP to the purified complexes, followed by incubation at room temperature for 5 minutes. To solve the orientation preference problem to the greatest extent, either CHAPSO or fluorinated octyl maltoside (FOM) was added immediately before sample vitrification (Fig. 1b). Three datasets, namely, p97-Npl4/Ufd1-Ub ${ }_{n}$-Eos-CHAPSO, p97-Npl4/Ufd1-Ub - -Eos-FOM and $\mathrm{p} 97-\mathrm{Npl} 4 / \mathrm{Ufd} 1-\mathrm{Ub}_{6}-\mathrm{FOM}$, were collected, and a total of nine different cryo-EM maps with resolutions ranging from 2.9 to $3.8 \AA$ were reconstructed using single-particle analysis (Supplementary Figs. 1-3, Tables 1 and 2 and Supplementary Table 1). Atomic models were built on the basis of existing p97 structures (Tables 1 and 2 and Supplementary Table 1). For well-resolved regions, individual residues were manually built into the density. For flexible regions, rigid-body docking was used to fit individual secondary structure elements or subdomains (see Methods for details).
Open and closed states of the D2 ring. In the p97-Npl4/ Ufd1- $\mathrm{Ub}_{n}$-Eos-CHAPSO dataset, two conformational states of the $\mathrm{D} 2$ ring were resolved (Fig. 1c,d). In the open state $(3.6 \AA$, Fig. 1c, corresponding to class 2 in Supplementary Fig. 1), all six D2 domains interacted with the substrate and formed an open, right-handed spiral staircase, whereas in the closed state (3.1 A, Fig. 1d, corresponding to class 1 in Supplementary Fig. 1), the bottom D2 domain (termed chain F) detached from the substrate and shifted up to interact with the top D2 domain (termed chain E), closing the gap of the spiral in the open state. Only five D2 domains (chains A-E) interacted with the substrate in the closed state. Overlay of these two states suggested a 'power-stroke'-like motion of one D2 domain, which detached from the substrate in the closed state and reattached to the substrate in the open state (Supplementary Video 1). This motion is responsible for the translocation of the unfolded peptide. Compared to the same complex structure in the presence of ATP $\gamma S$ but without the substrate ${ }^{26}$ (PDB 7JY5), there was a relative 


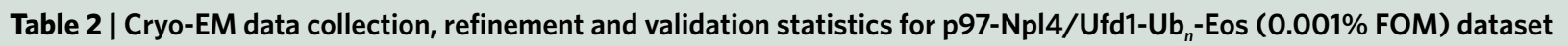

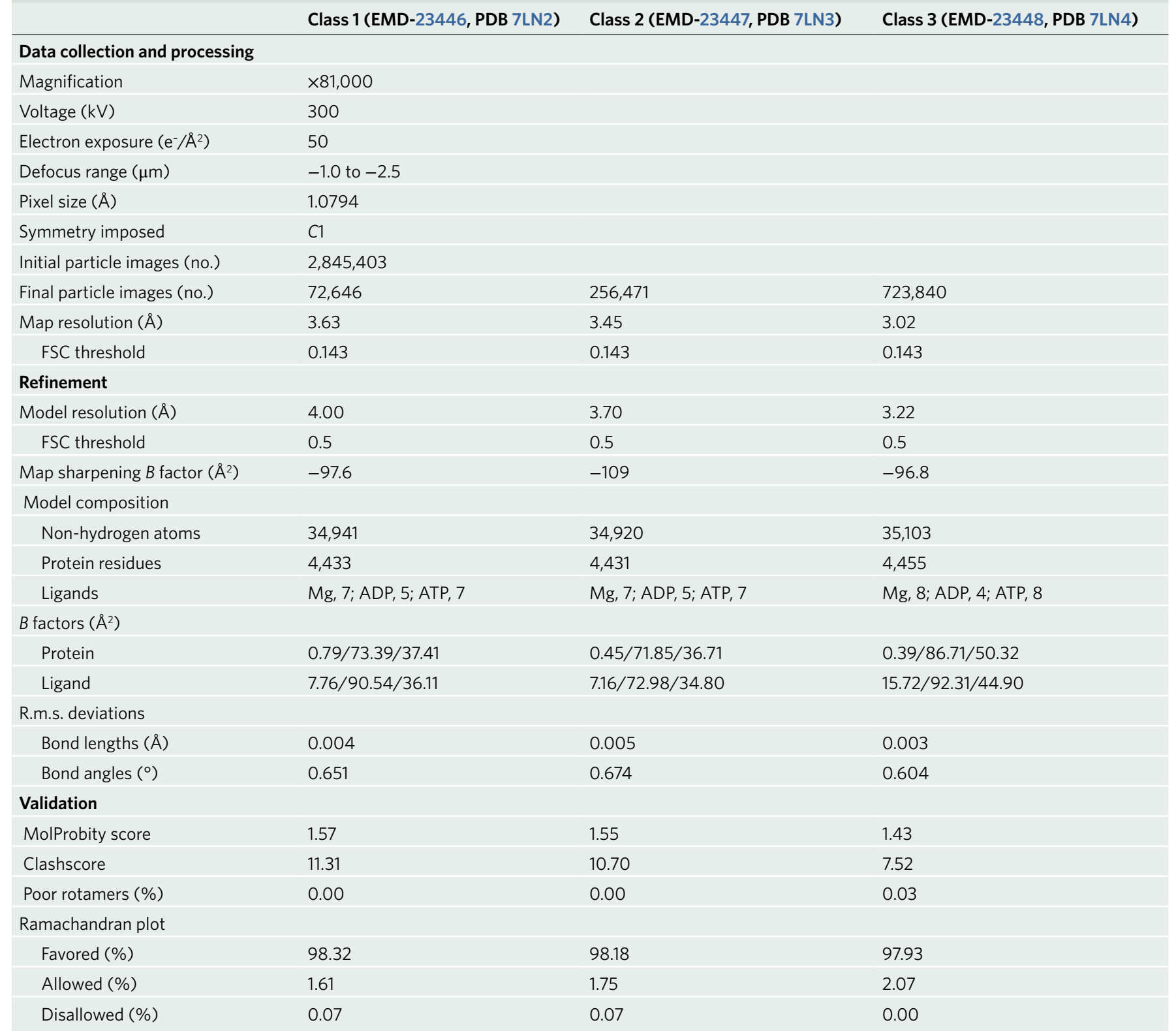

20-degree rotation of the D2 ring to the D1 ring in both states (Fig. 1e and Extended Data Fig. 2a). A superimposition of the two states showed that the D2 domain immediately above chain F (chain A) also shifted up from the open state to the closed state (Extended Data Fig. 3a and Supplementary Video 1). The particles in the open state were substantially less than those in the closed state (13\% versus $70 \%$ ), suggesting that the open state is relatively transient. A third class was resolved from the three-dimensional (3D) classification (3.8 $\AA$, class 3 in Supplementary Fig. 1), with better resolved density for the cofactor Npl4 but otherwise the same conformation as the closed state.

Conformational changes in the D1 ring. In the p97-Npl4/ Ufd1- $\mathrm{Ub}_{n}$-Eos-FOM dataset, three conformational states of the D1 ring were resolved (Supplementary Fig. 2). All the D2 rings were in the same conformation as the closed state described above. Using the same naming convention, we discovered from the superimposed structures that chain A shifted up slightly from class 1 (3.6 $)$ to class $2(3.4 \AA)$ and much further from class 2 to class $3(3.0 \AA)$
(Extended Data Fig. 4a). No open spiral conformation similar to the open state of the D2 ring was observed for the D1 ring, although a right-handed spiral staircase was still formed by chains B-F. The three states of the D1 ring also suggested a power-stroke-like motion despite the differences from the D2 ring (Supplementary Video 2). It is worth noting that no conformational changes in the D1 ring were observed for the $\mathrm{p} 97-\mathrm{Npl} 4 / \mathrm{Ufd} 1-\mathrm{Ub}_{n}$-Eos-CHAPSO dataset.

Sequential but asynchronous hydrolysis in D1 and D2 rings. The resolutions of the maps allowed us to build atomic models and identify the nucleotides in most nucleotide-binding sites (Extended Data Figs. 3d and 4d). For the less well-resolved chains A and F, the identity of the nucleotides could not be determined with certainty, but the density of a nucleotide was clearly visible (Extended Data Figs. 3d and 4d). All nucleotide-binding sites were occupied, suggesting that either the nucleotide exchange was very fast, or the mutations used for structure determination prevented the capture of an apo state. When superimposing all the AAA domains within the same ring, two major conformations were observed: one with 


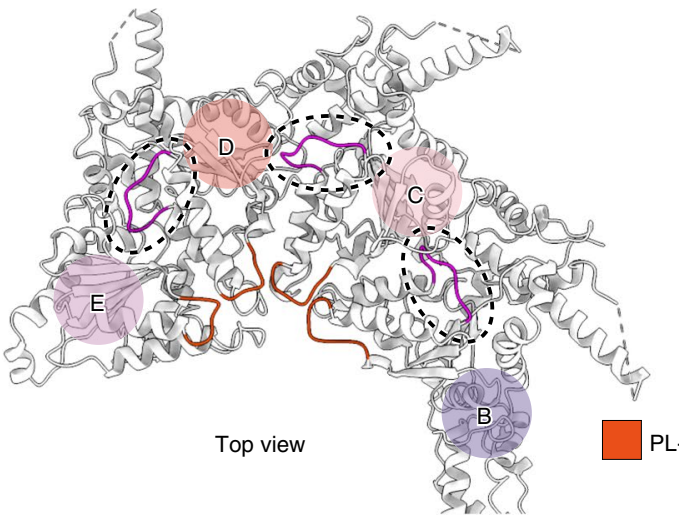

b

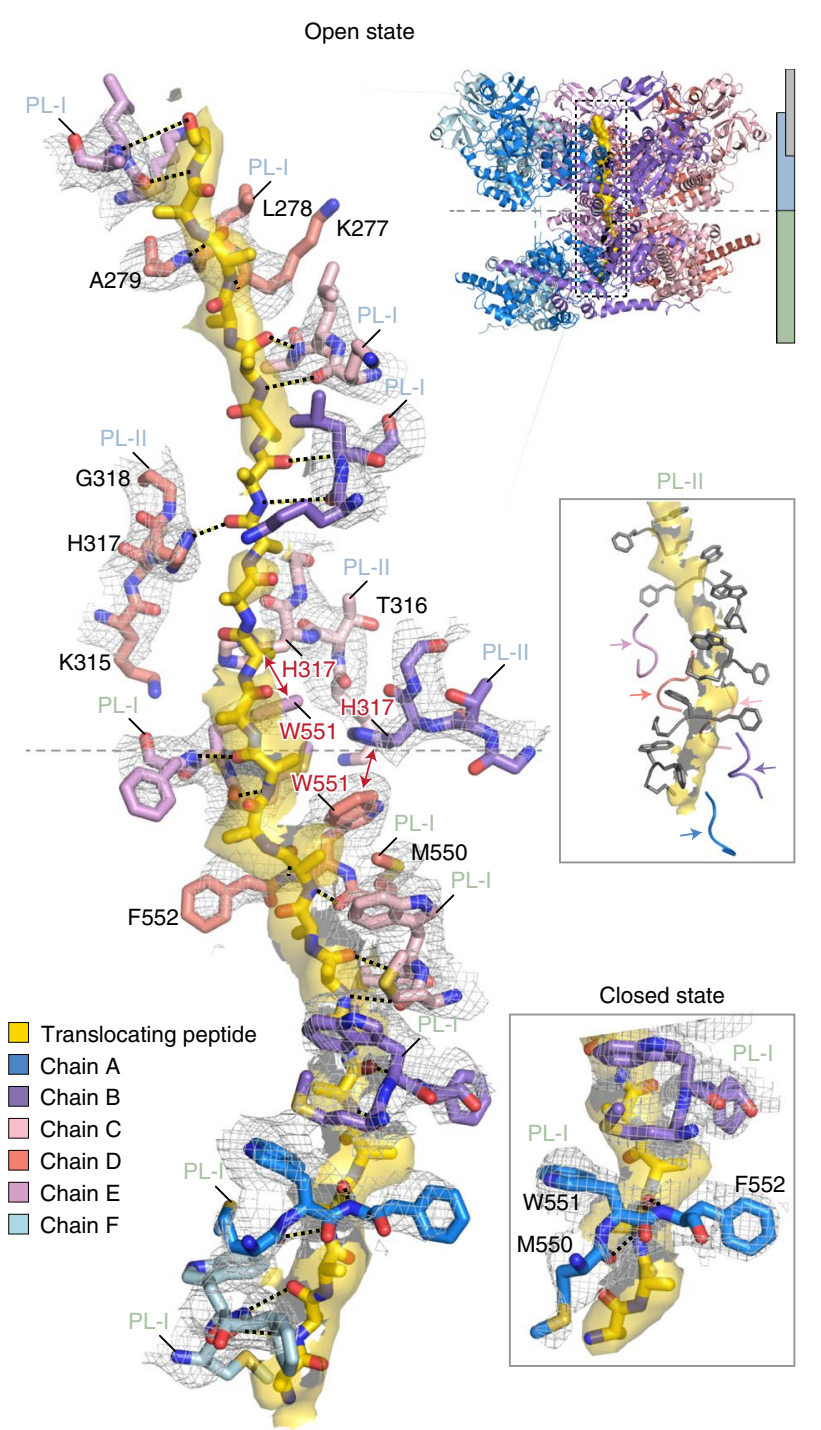

PL-I

D2 ring closed state

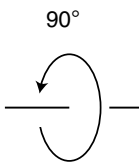

$90^{\circ}$

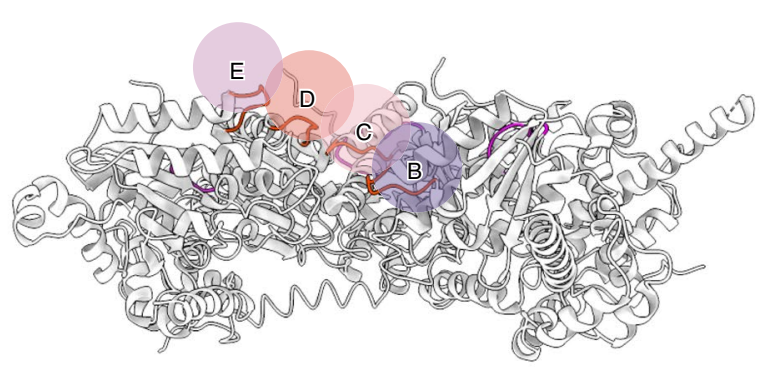

Side view c

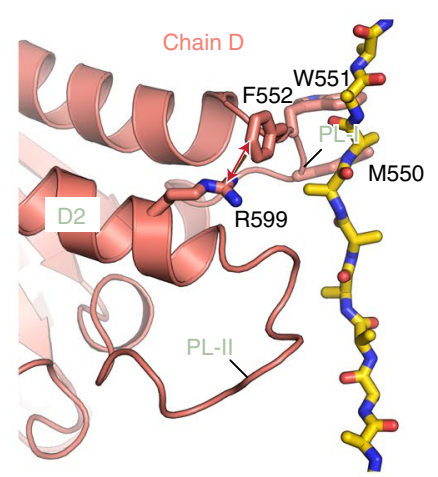

d

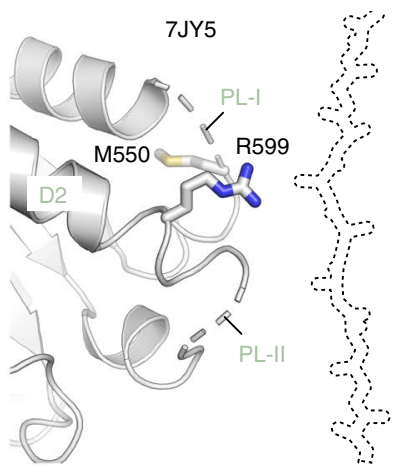

f

$\mathrm{p} 97+\mathrm{Ub}_{6}+\mathrm{FOM}$

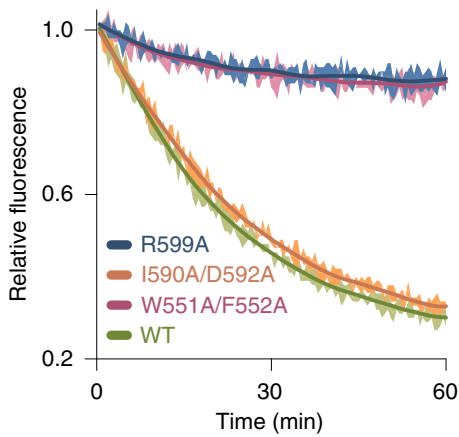

Translocating peptide only observed for D2 ring

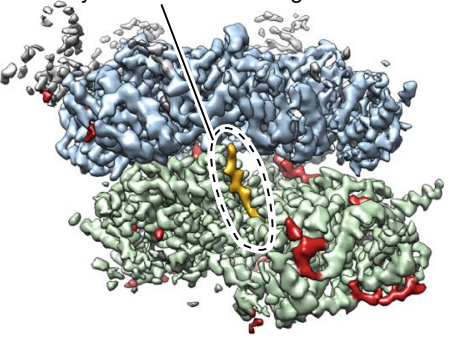

g

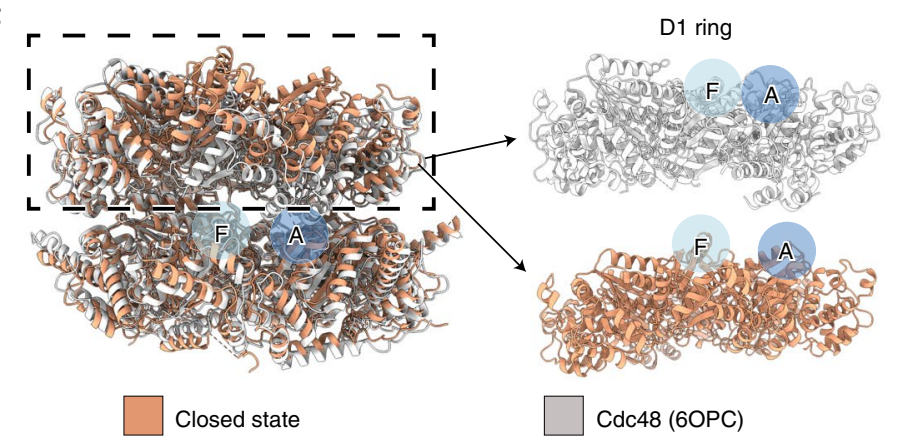

Fig. 2 | Interactions between the pore loops and the substrate. a, Illustrations showing the arrangement of PL-I and ISS motif in chains B-E in the D2 ring of the closed state. $\mathbf{b}$, Interactions between the translocating peptide and the interacting pore loops in the D1 and D2 rings. The density of the pore loops and the translocating peptide are shown as a gray mesh and yellow surface, respectively. Contour level: 6.0 root mean square deviation (r.m.s.d.). The closed state and PL-II of the D2 ring are shown in the insets. Hydrogen bonds between the pore loops and the peptide are labeled with black dotted lines. $\pi-\pi$ interactions between H317 and W551 are labeled with red double arrows. c,d, A comparison of the D2 domain in chain D of the open state (c) and that of the nontranslocating structure (d, PDB 7JY5). The D2 domains are aligned. e, Substrate unfolding assay for the pore loop mutants in the D2 domain of p97. The curves are presented as mean values \pm s.d. from triplicate experiments. WT, wild type. f, Unsharpened cryo-EM map (class 1) of the p97-Npl4/Ufd1$\mathrm{Ub}_{6}$-FOM dataset (Supplementary Fig. 3). The map is shown in a similar orientation to that in Fig. 1d,e. Contour level: 0.015. g, A comparison of cryo-EM models of translocating structures of p97 (closed state, orange) and Cdc48 (PDB 6OPC, light gray). The two models were overlayed on the basis of the D2 ring. Right, separate views of the D1 rings (boxed area) after the overlay. Positions of chains A and F are marked. Source data for $\mathbf{e}$ are available online. 
a larger angle between the $\alpha / \beta$ - and the $\alpha$-subdomain, potentially corresponding to an ATP-binding conformation, and the other with a smaller angle between the $\alpha / \beta$ - and the $\alpha$-subdomain, potentially corresponding to an ADP-binding conformation (Extended Data Figs. 3c and 4c). Such binary conformations have been observed for other substrate-engaged AAA proteins ${ }^{15-19,29,30}$.

When focusing on the well-resolved sites, we discovered a new compact ATP-binding mode in both the $\mathrm{D} 1$ and $\mathrm{D} 2$ rings involving a previously defined ISS motif ${ }^{31}$. Specifically, the ISS motif changed from a helical conformation to a triangular loop that inserted into the neighboring nucleotide-binding site (Fig. 1e,f). These conformational changes were closely related to the spiral staircases formed by the pore loops (Fig. 2a and Extended Data Fig. 5a,b). The L335 in the D1 domain and M611 in the D2 domain are the key residues interacting with the hydrophobic residues in the Walker B motifs of the neighboring subunits (Fig. 1f). As a result, ATP is coordinated by three more residues than the ATP $\gamma$ S-bound p97-Ufd1-Npl4 complex (PDB 7JY5), including one additional arginine finger residue (R362 in D1 and R638 in D2), an aspartic acid from the ISS motif (D333 in D1 and D609 in D2) and a basic residue following the Walker A motif (R256 in D1 and K529 in D2) (Fig. 1f). This binding mode was observed only when ATP was engaged and only for two to four consecutive binding sites in both rings (Fig. 1e and Extended Data Figs. $3 \mathrm{~b}$ and $4 \mathrm{~b}$ ). We hypothesize that this tight binding mode is required for the rotational compression of ATPase rings, which leads to the formation of spiral staircases and is essential for substrate translocation. For ATP hydrolysis and ADP release to occur, the ISS motif needs to be retracted to loosen the binding with the nucleotide. For example, the ISS motif in the D2 domain of chain A retracted in the closed state (not in the open state), since the neighboring D2 domain in chain B is the next subunit to undergo ATP hydrolysis and ADP release (Fig. 1e and Extended Data Fig. 3b).

Another unexpected observation is that the hydrolysis in the D1 and D2 rings is not synchronized, which means that at a given time the D1 and D2 domains of the same chain may engage different nucleotides. If viewed from the top, hydrolysis propagates counterclockwise in both ATPase rings, with the D1 ring one or two subunits ahead of the D2 ring (Fig. 1e). This feature is unique for human $\mathrm{p} 97$ and has not been observed for other type II AAA proteins, including yeast $\mathrm{Cdc} 48$. It is possible that the mutations used for structure determination caused the asynchrony. Further studies are necessary to explain the observation.

Interactions between the pore loops and the substrate. In all six maps discussed above, we observed the density corresponding to a translocating peptide going through the central pore of $\mathrm{p} 97$ (Fig. 2b). A continuous extended peptide could be modeled into the density. The peptide went through the pore from the $\mathrm{N}$ terminus (bottom in Fig. 2b) to the C terminus (top in Fig. 2b), as the other direction fit much worse into the density. This directionality of translocating peptide is consistent with that of Cdc48 (refs. ${ }^{18,19}$ ). Since the density corresponding to the side chains was averaged, polyalanine was modeled.

In general, the density of the translocating peptide in the D2 ring was better than that in the D1 ring. Three residues in pore loop I (PL-I) of the D2 domain, namely, M550, W551 and F552, interacted with the peptide like a 'pitching grip' (Fig. 2b). Each PL-I formed two hydrogen bonds with the nitrogen and carbonyl oxygen of one residue in the translocating peptide, through the carbonyl oxygen of M550 and nitrogen of F552. Every other residue in the translocating peptide was hydrogen bonded by a PL-I, so 12 residues were engaged with six PL-I in the D2 ring, which formed a spiral in the open state, with chain $\mathrm{F}$ at the bottom and chain $\mathrm{E}$ at the top (Fig. 2b). In the closed state, the PL-I of chain F detached from the translocating peptide. The remaining five PL-I formed the spiral in a very similar way except for the side chain of M550 in chain A (now the bottom chain), which was pointing downwards and would not allow an additional PL-I to engage (Fig. 2b, inset). In both the open and closed states, the densities of the five PL-II in the D2 ring were resolved (chains A to E) but at lower resolution (Fig. 2b, inset). PL-II in the D2 ring did not form hydrogen bonds with the translocating peptide and likely played a less important role in translocation. We mutated the residues of PL-I and PL-II and performed the substrate unfolding assay ${ }^{1,26,32}$. Indeed, the PL-I mutation (W551A/F552A) abolished the unfolding activity, whereas the PL-II mutation (I590A/ D592A) showed no effect (Fig. 2e). A closer examination revealed that R599 was $\pi$-stacked with F552 of PL-I (Fig. 2c). This interaction might be critical to stabilize the 'pitching grip' conformation of PL-I, since the R599A mutant did not show any unfolding activity, similar to the PL-I mutation (Fig. 2e). In contrast, the pore loops in the D2 domain of the nontranslocating structure (PDB 7JY5) were mostly unstructured (Fig. 2d).

PL-I in the D1 ring interacted with the translocating peptide in the same fashion as that in the D2 ring, except that only four PL-I (chains B-E) were engaged and the three residues were K277, L278 and A279 (Fig. 2b). The 'pitching grip' and hydrogen-bonding pattern still existed, but the interaction was not as strong as that of the D2 ring, since the average length of the hydrogen bonds (measured from nitrogen to oxygen) was $3.9 \AA$ in the D1 ring and $3.4 \AA$ in the D2 ring. Two PL-II of the D1 ring (chains C and D) were involved in the interaction with the peptide by forming a hydrogen bond through H317 (Fig. 2b). Interestingly, H317 of chains B and C also stacked with W551 (PL-I of D2) of chains D and E, respectively (Fig. $2 \mathrm{~b}$, red double arrow). This $\pi-\pi$ stacking might be involved in the coordination of two ATPase rings.

To further investigate the behavior of p97 with substrates of different sizes, we used a much smaller substrate, $\mathrm{Ub}_{6}$ (Extended Data Fig. 1c), and collected a third dataset, p97-Npl4/Ufd1-Ub ${ }_{6}-\mathrm{FOM}$ (Supplementary Fig. 3). Again, three conformations of the D1 ring that were very similar to those of the p97-Npl4/Ufd1-Ub ${ }_{n}$-Eos-FOM dataset were resolved. Intriguingly, the density corresponding to the translocating peptide was resolved only in the D2 ring (Fig. 2f). The D1 ring was essentially void of the translocating peptide despite adopting the same conformation. One possible explanation is that the D1 ring was engaged at different processing states depending on the topology of the substrates used for structure determination. Nevertheless, mutations in pore loops of the D1 domain affected the unfolding activity (Extended Data Fig. 1d), suggesting that the D1 domain also plays a role in substrate translocation.

We noticed that the $\mathrm{D} 2$ ring of the closed state is similar to those of $\mathrm{Cdc} 48-\mathrm{ADP} \cdot \mathrm{BeF}_{x}$ structures $^{18,19}$, all of which have five PL-I engaging with the translocating peptide. When the D2 rings from these structures were superimposed, it was obvious that the two rings of p97 were out of register, that is, the lowest D2 and D1 domains are not in the same chain, whereas in Cdc48 the two rings essentially formed a single 'split washer' (Fig. $2 \mathrm{~g}$ and Extended Data Fig. 5c,d). Accordingly, the entire chain F (named on the basis of Fig. 1) is flexible in previous $\mathrm{Cdc} 48-\mathrm{ADP} \cdot \mathrm{BeF}_{x}$ structures, whereas in our structures the D2 domain of chain $\mathrm{F}$ and the D1 domain of chain A are the most flexible. These features are consistent with the asynchronous hydrolysis occurring in the D1 and D2 rings.

$\mathrm{N}$ - and C-terminal tails and the linker between ATPase rings. In addition to the ATPase rings, some of the $\mathrm{N}$ - and $\mathrm{C}$-terminal tails (Fig. 1a) of p97 were also resolved in the cryo-EM maps. Both the resolved N-terminal (L12 to R22) and C-terminal (G767 to S775) tails interacted with the neighboring subunit in a counterclockwise manner if viewed from the top (N-terminal tail, Fig. 3a), or a clockwise manner if viewed from the bottom (C-terminal tail, Fig. 3c). The N-terminal tail interacted with an acidic patch in the $\alpha$-subdomain of the D1 domain (Fig. 3b). The C-terminal tail interacted with a groove in the $\alpha$-subdomain of the D2 domain 


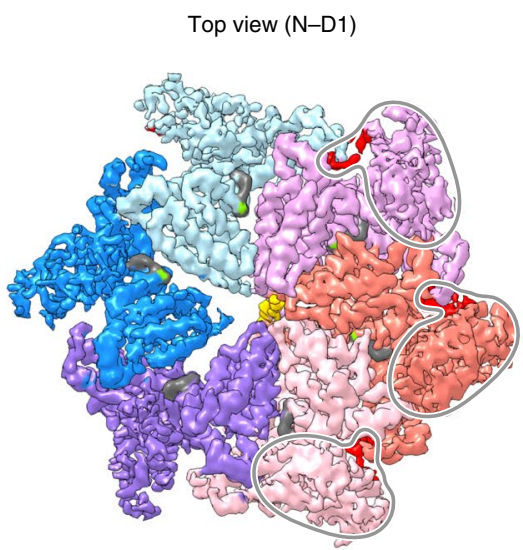

c

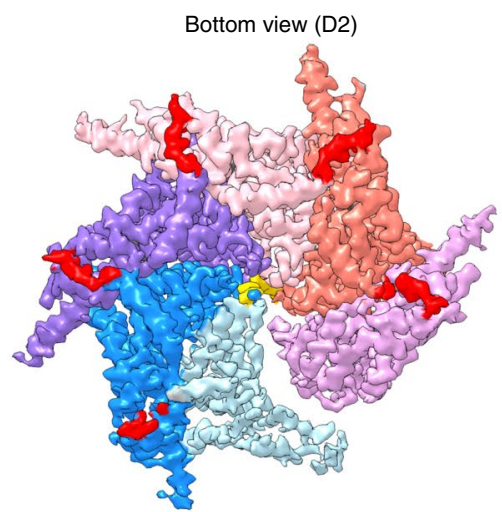

b

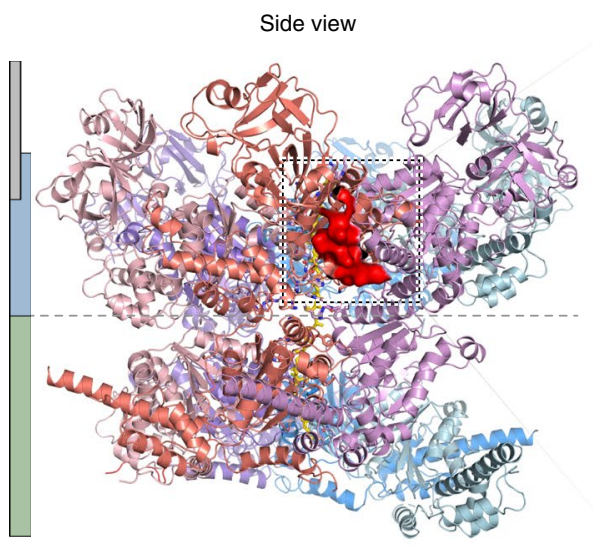

d

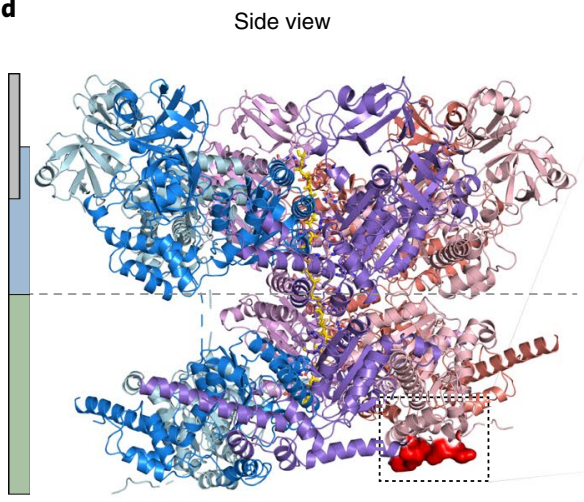

e

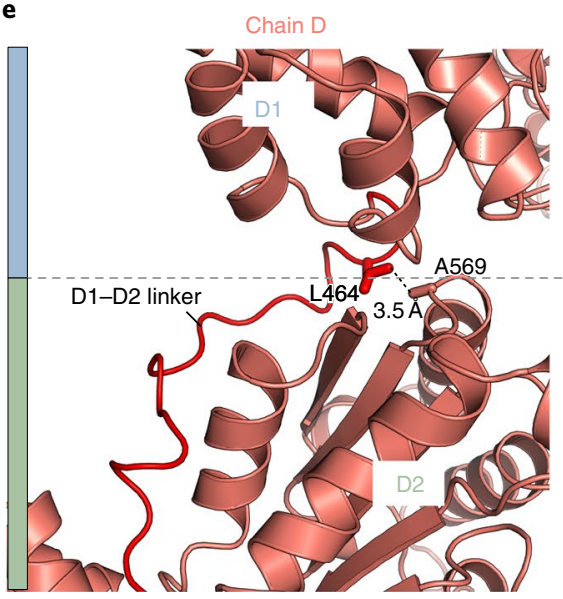

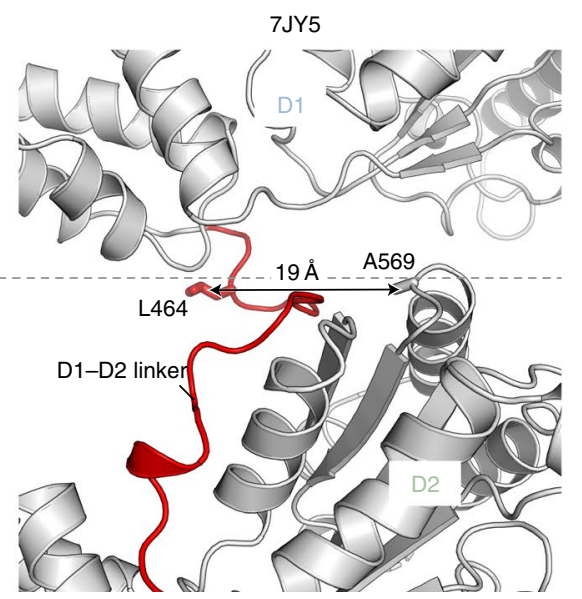
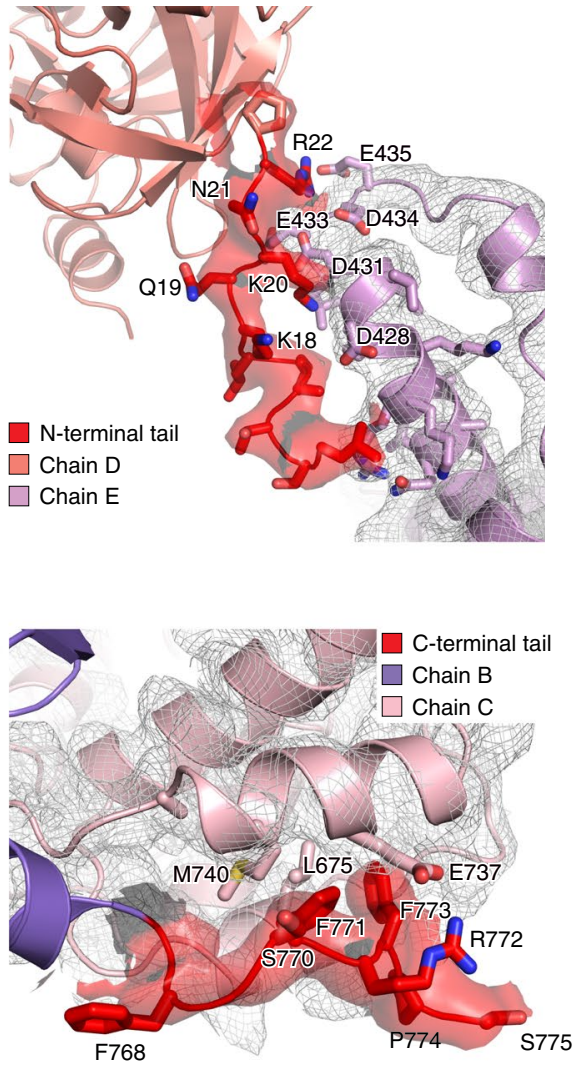

$\mathbf{f}$

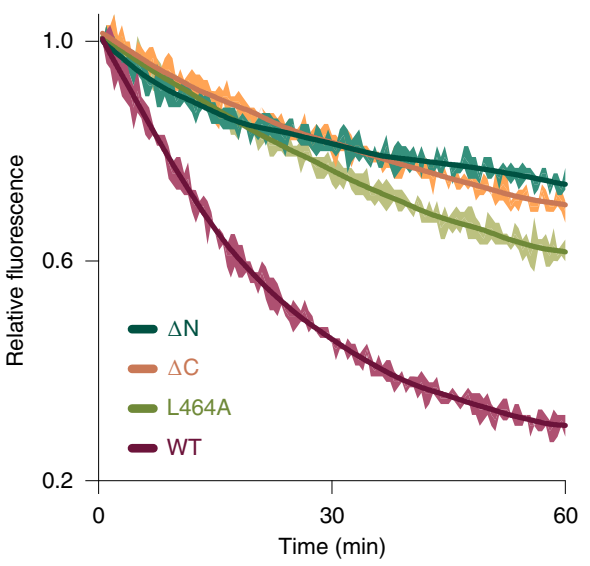

Fig. 3 | N- and C-terminal tails and the linker between the D1 and D2 rings. a, Top view of the unsharpened cryo-EM map focused on the N and D1 domains (open state). N-terminal tails are colored in red. Contour level: 0.015. b, Side view of the cryo-EM model of the open state with the density of one N-terminal tail highlighted and magnified. Contour level: 6.0 r.m.s.d. c, Bottom view of the unsharpened cryo-EM map focused on the D2 ring (open state). C-terminal tails are shown in red. Contour level: 0.015. d, Side view of the cryo-EM model of the open state with the density of one C-terminal tail highlighted and magnified. Contour level: 6.0 r.m.s.d. e, A comparison of the D1-D2 linker in chain D of the open state and that of the nontranslocating structure (PDB 7JY5). The D2 domains are aligned. f, Substrate unfolding assay for tail and linker mutants of p97. The curves are presented as mean values \pm s.d. from triplicate experiments. Source data for $\mathbf{f}$ are available online.

(Fig. 3d). Such interactions have not been described before and may play important roles in the coordination between different chains. Truncations of either the $\mathrm{N}$ - or C-terminal tails greatly affected the unfolding activity of p97 (Fig. 3f). The linker between the D1 and $\mathrm{D} 2$ rings was resolved for chains $\mathrm{B}-\mathrm{E}$. Compared to the structure of nontranslocating p97 (PDB 7JY5), a 19- $\AA$ shift of L464 was observed (Fig. 3e). In the translocating structure, L464 interacted with A569 in the D2 domain, which contributed to the relative twist of the D2 ring (Fig. 1e). The L464A mutation decreased the unfolding activity of p97 (Fig. 3f). Notably, the N- and C-terminal tails and the linker regions are less conserved between human p97 and yeast Cdc48, which might contribute to the different conformations of the ATPase rings.

NMS-873 inhibits the translocation by locking the ISS motif. Inhibition of $\mathrm{p} 97$ has emerged as a promising strategy in anticancer 
a<smiles>Cc1cc(OCc2nnc(SC3CCCC3)n2-c2cccnc2)ccc1-c1ccc(S(C)(=O)=O)cc1</smiles>
b

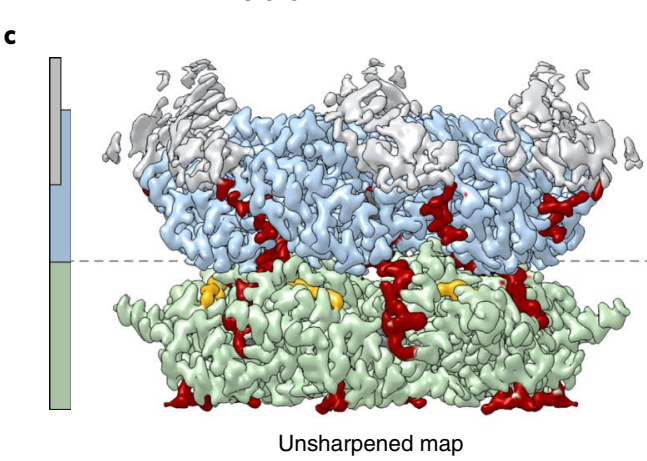

$\mathrm{N}$ domain

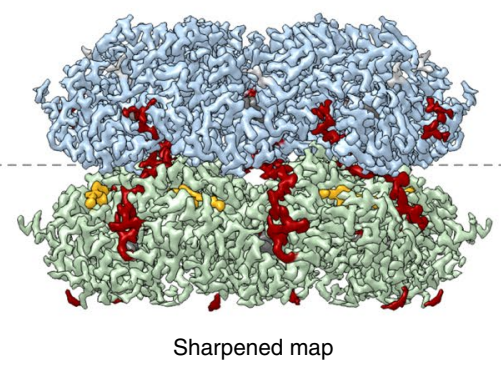

D1 domain
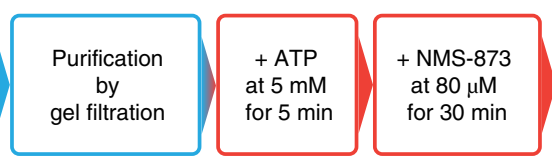

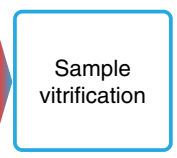

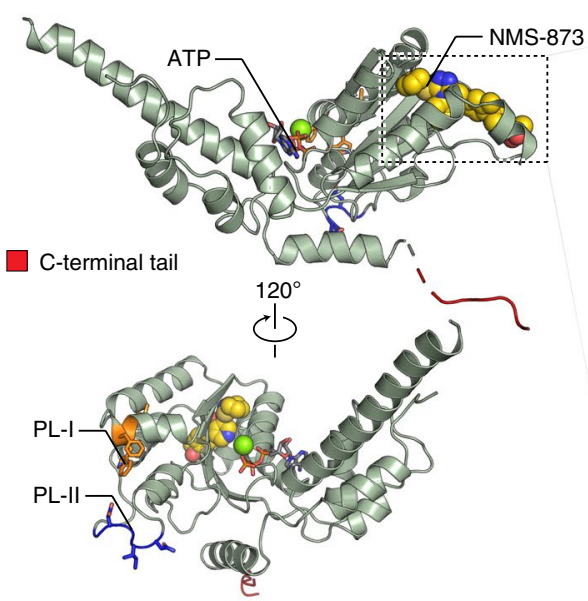

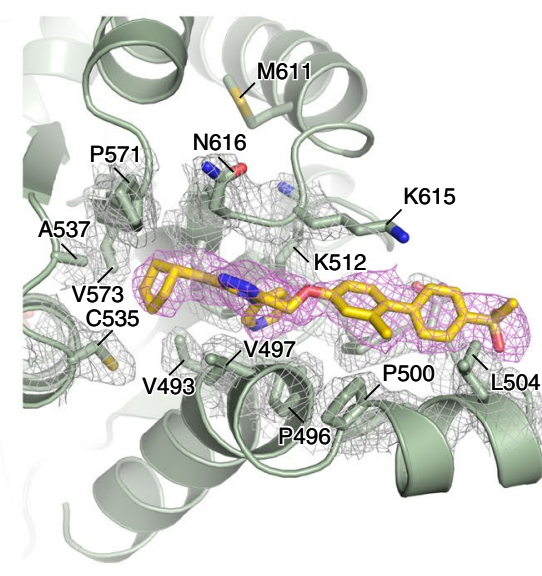

f
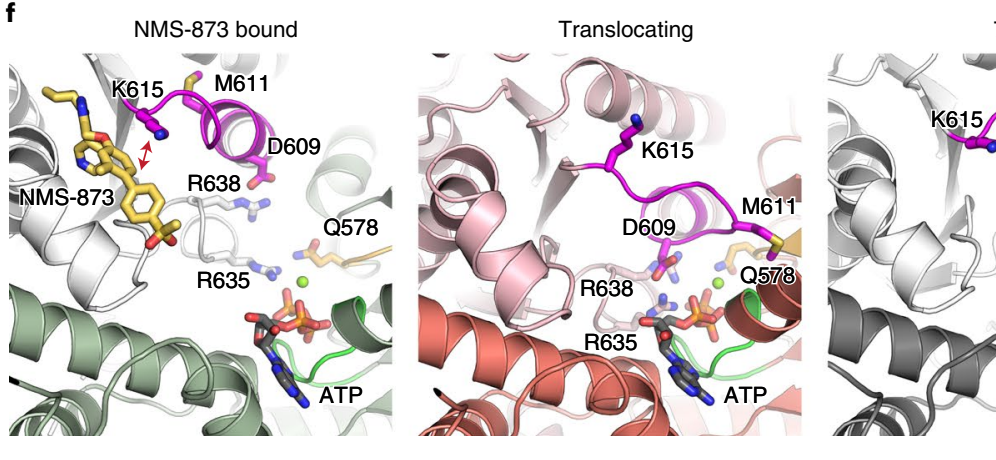

$\square$ NMS-873

Walker A motif

Walker B motif

$\square$ ATP $\gamma \mathrm{S}$
7JY5

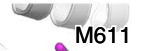

$90^{\circ} \quad 90^{\circ}$

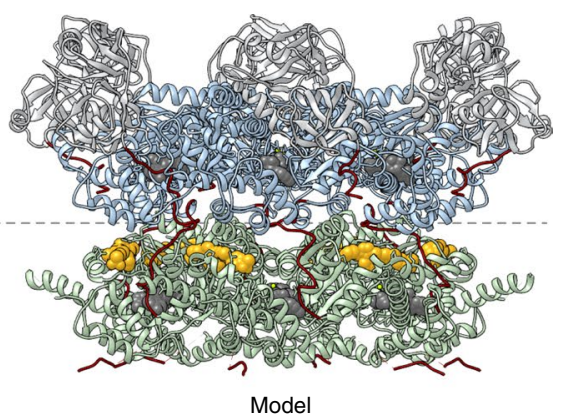

NMS-873 $\square$ ATP
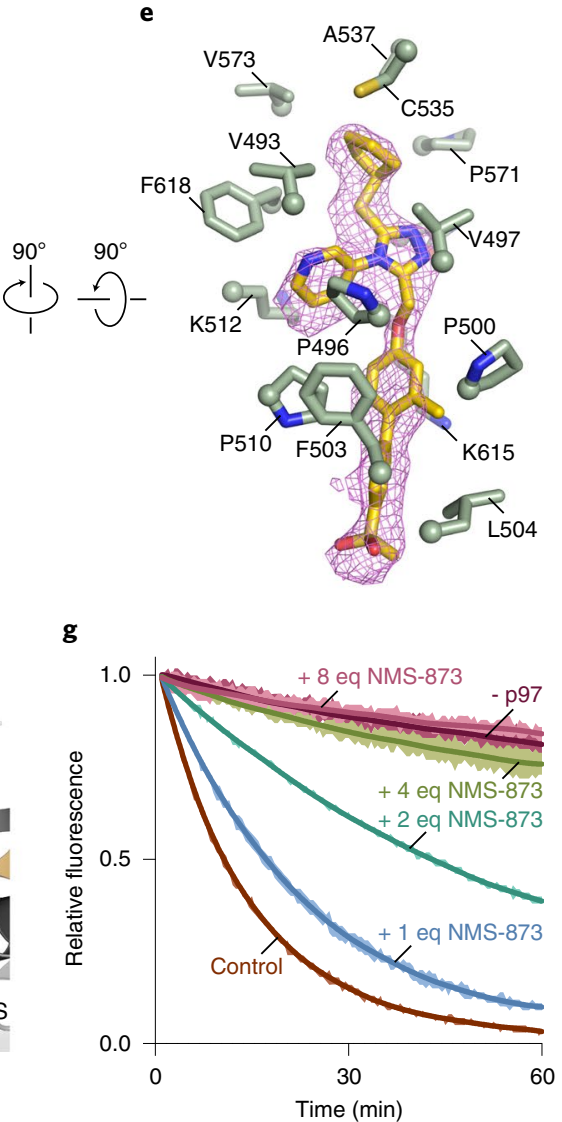

Fig. 4 | NMS-873 inhibits translocation by locking the ISS motif. a, Chemical structure of NMS-873. b, Sample preparation procedures. Steps performed at $4{ }^{\circ} \mathrm{C}$ are in blue boxes. Steps performed at room temperature are in red boxes. c, Cryo-EM maps and models of human p97 in complex with NMS873. The maps and models are aligned. Contour level: unsharpened map, 0.018; sharpened map, 0.052. $\mathbf{d}$, D2 domain of the complex structure in $\mathbf{c}$, with a zoom-in of the NMS-873-binding site on the right. The density of NMS-873 and the interacting residues are shown as magenta and gray mesh, respectively. Contour level: 6.0 r.m.s.d. e, A different view of the NMS-873-binding site, with side chains of interacting residues shown. Contour level: 6.0 r.m.s.d. f, A comparison of nucleotide-binding sites in NMS-873-bound, translocating (chain D in open state) and nontranslocating (PDB 7JY5) structures. The bottom D2 domains (green, salmon and gray) are aligned. $\mathbf{g}$, Substrate unfolding assay for wild-type p97 in the presence of different concentrations of NMS-873. One $p 97$ hexamer can bind 6 equivalents (eq) of NMS-873. The curves are presented as mean values \pm s.d. from triplicate experiments. Source data for $\mathbf{g}$ are available online.

and antiviral studies ${ }^{8}$. NMS-873, a previously discovered allosteric inhibitor of p97 (Fig. 4a), was recently characterized as a candidate for the treatment of cancers, neurodegenerative diseases and viral infections ${ }^{22-24}$. However, the mechanism of inhibition remained unclear. We introduced NMS-873 at saturation $(80 \mu \mathrm{M})$ after triggering ATP hydrolysis and substrate translocation (Fig. 4b). 


\begin{tabular}{|c|c|c|}
\hline & $\begin{array}{l}\text { p97-Npl4/Ufd1-Ub }- \text {-NMS-873 }(80 \mu M) \\
(0.001 \% \text { FOM) (EMD-23442, PDB 7LMY) }\end{array}$ & $\begin{array}{l}\text { p97-Npl4/Uf } \\
\text { (4 mM CHAP }\end{array}$ \\
\hline \multicolumn{3}{|l|}{ Data collection and processing } \\
\hline Magnification & $\times 81,000$ & $\times 81,000$ \\
\hline Voltage (kV) & 300 & 300 \\
\hline Electron exposure $\left(\mathrm{e}^{-} / \AA^{2}\right)$ & 50 & 50 \\
\hline Defocus range $(\mu \mathrm{m})$ & -1.0 to -2.5 & -1.0 to -2.5 \\
\hline Pixel size $(\AA)$ & 1.063 & 1.0794 \\
\hline Symmetry imposed & C6 & C6 \\
\hline Initial particle images (no.) & 782,499 & $1,266,504$ \\
\hline Final particle images (no.) & 372,419 & 501,204 \\
\hline Map resolution $(\AA)$ & 2.41 & 2.87 \\
\hline FSC threshold & 0.143 & 0.143 \\
\hline \multicolumn{3}{|l|}{ Refinement } \\
\hline Model resolution $(\AA)$ & 2.56 & \\
\hline FSC threshold & 0.5 & \\
\hline Map sharpening $B$ factor $\left(\AA^{2}\right)$ & -58.8 & \\
\hline \multicolumn{3}{|l|}{ Model composition } \\
\hline Non-hydrogen atoms & 35,646 & \\
\hline Protein residues & 4,470 & \\
\hline Ligands & Mg, 12; NM3, 6 (NMS-873); ATP, 6 & \\
\hline \multicolumn{3}{|l|}{$B$ factors $\left(\AA^{2}\right)$} \\
\hline Protein & $1.58 / 57.10 / 22.25$ & \\
\hline Ligand & $2.92 / 28.45 / 15.85$ & \\
\hline \multicolumn{3}{|l|}{ R.m.s. deviations } \\
\hline Bond lengths $(\AA)$ & $0.003(0)$ & \\
\hline Bond angles $\left({ }^{\circ}\right)$ & $0.845(3)$ & \\
\hline \multicolumn{3}{|l|}{ Validation } \\
\hline MolProbity score & 1.79 & \\
\hline Clashscore & 8.11 & \\
\hline Poor rotamers (\%) & 1.89 & \\
\hline \multicolumn{3}{|l|}{ Ramachandran plot } \\
\hline Favored (\%) & 97.29 & \\
\hline Allowed (\%) & 2.71 & \\
\hline Disallowed (\%) & 0.00 & \\
\hline
\end{tabular}

Two datasets were collected using $\mathrm{Ub}_{n}$-Eos and $\mathrm{Ub}_{6}$ as the substrate. A single map was obtained from each dataset with overall resolutions of $2.4 \AA\left(\mathrm{Ub}_{6}\right.$, Supplementary Fig. 4 and Table 3$)$ and $2.9 \AA$ $\left(\mathrm{Ub}_{n}\right.$-Eos, Supplementary Fig. 5 and Table 3$)$. The two maps are essentially identical; therefore, only the map with the higher resolution is shown (Fig. 4c). Unexpectedly, both maps showed that p97 maintained a six-fold symmetry and no translocating peptides were found in the central pore, although a substrate was present and ATP hydrolysis was triggered before adding NMS- 873 . In the six-fold symmetric structure of $\mathrm{p} 97, \mathrm{NMS}-873$ binds at a cryptic groove next to the ISS motif of the D2 domain (Fig. 4d) and is surrounded by many hydrophobic residues (Fig. 4e). A critical interaction was observed between K615 in the ISS motif and the biphenyl group of NMS-873 (Fig. 4d). As a result, the ISS motif is locked and prevented from essential conformational changes discovered in the translocating structures (Fig. 4f). The pore loops in the D2 domain of the NMS-873-bound structure were resolved but retracted from the pore compared to the translocating structure (Extended Data Fig. 2e). Together, these structural changes prevented the formation of a spiral staircase by the pore loops of the D2 ring.

Compared to the ATP $\gamma S$-bound nontranslocating structure (PDB 7JY5), the relative positions of the ATPase rings in the NMS873-bound structure are similar (Extended Data Fig. 2b). The difference is that the bottom opening of the D2 ring is enlarged, which is similar to that of the ADP-bound structures ${ }^{13}$, although ATP was engaged (Extended Data Fig. 2c). The smaller angle between the $\alpha / \beta$ - and the $\alpha$-subdomains also suggested that the NMS-873-bound D2 ring is more similar to an ADP-bound state, indicating that NMS-873 can affect the conformation of the D2 ring regardless of the nucleotide states (Extended Data Fig. 2d). Superimposing the NMS-873-bound structure with another inhibitor-bound structure (UPCDC30245, PDB 5FTJ (ref. ${ }^{13}$ )) showed the similarity 


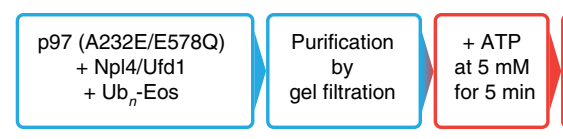

c

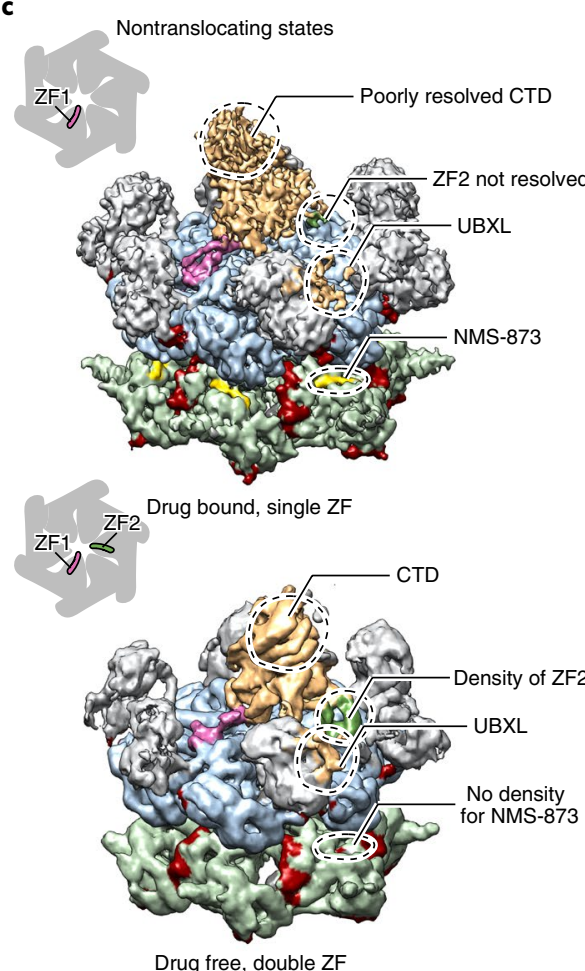

$\square$ N domain $\square$ D1 domain $\square$ D2 domain
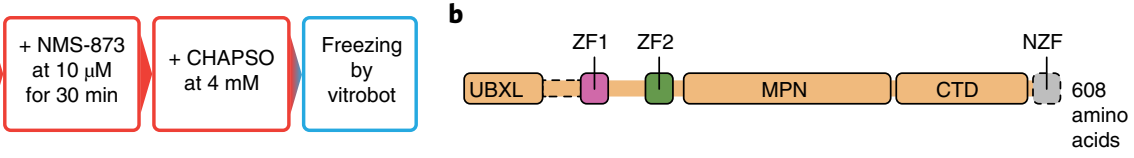

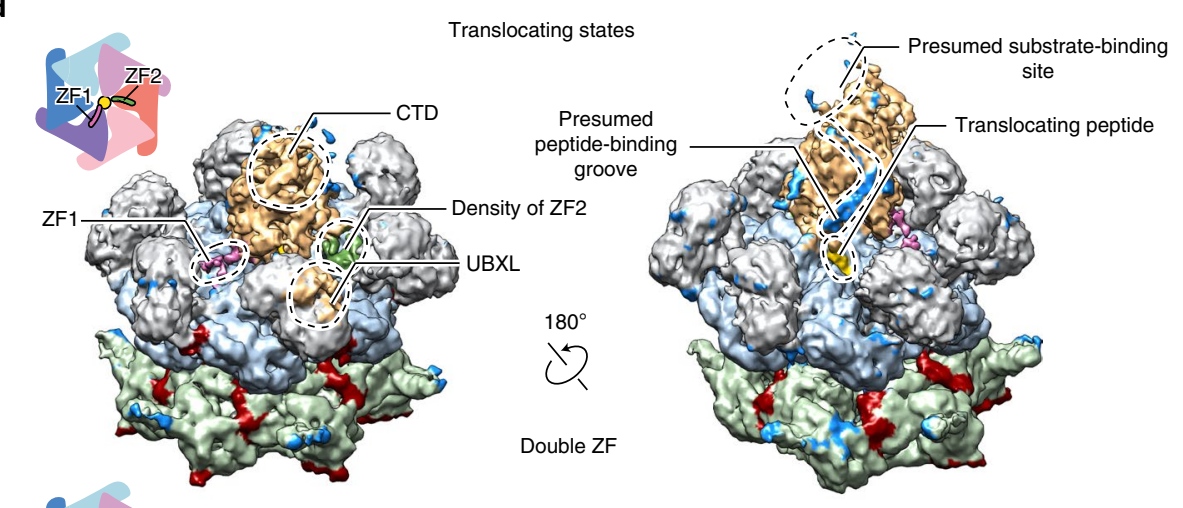

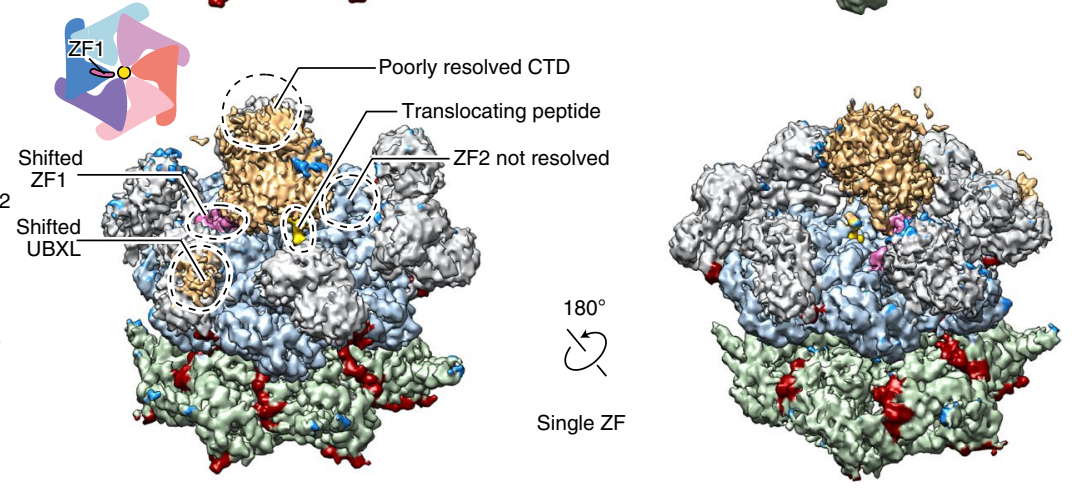

Fig. 5 | Conformational states of Npl4 in the presence of NMS-873 at a substoichiometric concentration. a, Sample preparation procedures. Steps performed at $4{ }^{\circ} \mathrm{C}$ are in blue boxes. Steps performed at room temperature are in red boxes. b, Domain architecture of human Npl4. The color code for the individual motifs is the same as in $\mathbf{c}$ and $\mathbf{d}$. The dotted parts are not resolved in the cryo-EM maps. c, Two unsharpened cryo-EM maps in nontranslocating states resolved from the dataset (Supplementary Fig. 6), one bound to NMS-873 (top). Contour level: 0.013. d, Two unsharpened cryo-EM maps in translocating states resolved from the dataset. Contour level: top, 0.01; bottom, 0.013. Different positions of zinc-finger motifs of Npl4 are illustrated in the insets.

in the conformations of the pore loops. The difference is that UPCDC30245 only shares part of the binding pocket of NMS-873 in the D2 domain (Extended Data Fig. 2f,g). In particular, the interaction between K615 and the biphenyl group of NMS-873 does not exist in the UPCDC30245-bound structure (Fig. 4f and Extended Data Fig. 2g), which was also reported from previous biochemical characterizations $s^{13,21}$. Combined with the previous finding that UPCDC30245 binds p97 only in the presence of ADP and acts as an uncompetitive inhibitor, whereas NMS-873 can bind p97 in the absence of nucleotides and acts as a noncompetitive inhibitor ${ }^{8}$, we concluded that the ISS locking mechanism of NMS-873 through the interaction with K615 is the key to the noncompetitive allosteric inhibition.

Conformational states of Npl4 in the presence of NMS-873. We further discovered that NMS-873 did not fully inhibit the unfolding activity of p97 at a substoichiometric concentration (6 equivalents is the stoichiometric concentration, Fig. $4 \mathrm{~g}$ ). With the reasoning that intermediate structures could be captured using NMS-873 as a modulator, a dataset was collected following the same protocol using $\mathrm{Ub}_{n}$-Eos as the substrate and in the presence of $10 \mu \mathrm{M}$ NMS873 ( $\sim 0.2$ equivalents, Fig. $5 \mathrm{a}$ ). As expected, multiple intermediate structures were resolved after 3D classification (Supplementary
Fig. 6 and Supplementary Table 2). Two out of six maps ( 11\% of particles) are in the nontranslocating state, with flat and symmetric ATPase rings and no density for a translocating peptide (Fig. 5c). One of the nontranslocating maps (class $1, \sim 8 \%$ of particles) showed the density of NMS-873 (Fig. 5c, top) in all six binding pockets, suggesting that the binding of NMS-873 is highly cooperative. The cooperativity of NMS-873 binding was also evident when we introduced the compound in the middle of the unfolding assay (Extended Data Fig. 1e). The cofactor Npl4 was resolved in four out of six maps (Supplementary Fig. 6). Both nontranslocating maps showed density for Npl4, with either one or two of its zinc-finger motifs (ZF1 and ZF2, Fig. 5b) binding on top of the D1 ring (Fig. 5c). Such seesaw conformations of Npl4 have been described in a previous study ${ }^{26}$. The four translocating structures all had spiral ATPase rings and a translocating peptide similar to the conformation of the closed state (Supplementary Fig. 6). Two maps (class 5 and class 6) had very poor densities of Npl4 and differed in the D1 ring in a similar way to class 1 and class 3 of the p97-Npl4/Ufd1-Ub - -Eos-FOM dataset. The other two maps showed density for Npl4, but with its zinc-finger motifs interacting with different chains (Fig. 5d), suggesting a rotation of Npl4 on top of the D1 ring. Although the resolution of Npl4 is not sufficient for atomic model building, a trace density connected to the 
translocating peptide can be seen to be engaged in a groove of Npl4 (Fig. 5 d, top), which is reminiscent of a substrate processing complex structure of Cdc48 (ref. ${ }^{19}$ ).

\section{Discussion}

Here, we report the cryo-EM structures of human p97 in several working states, with a translocating peptide going through the central pore. Together, the structures revealed power-stroke-like conformational changes in both the D1 and D2 rings. Surprisingly, the two AAA domains undergoing power-stroke motion in each ring were not from the same chain, which seemed to be a unique feature of human p97 and was not observed for Cdc48 and other type II AAA proteins ${ }^{15,16,18,19,29,30}$. The unusual structural feature corresponds to the asynchronous hydrolysis taking place in the two ATPase rings (Fig. 1e and Extended Data Fig. 5c,d). Regarding translocation, the D2 ring seemed to play a more important role by tightly interacting with the peptide through backbone hydrogen bonds. Previous biochemical studies have shown that the D2 ring of p97 had higher ATPase activity and provided the driving force for translocation, which is consistent with our structures ${ }^{33}$. The structures of Cdc48 also showed fewer interactions between the translocating peptide and the pore loops of the D1 ring ${ }^{19}$, further supporting this hypothesis. However, our substrate unfolding assay suggested that the D1 ring also played a role in translocation, likely through coordination with the D2 ring (Extended Data Fig. 1d).

Although the structures strongly support a sequential hydrolysis model $^{34}$, it is not clear whether the power strokes in the D1 and D2 rings take place concurrently. Multiple structures from a single dataset are needed to address this question. In this study, the motions of the D1 and D2 rings were resolved from two datasets, one using CHAPSO and the other using FOM as the detergent to fix the orientation preference. CHAPSO required a much higher concentration of the complex than FOM $(8 \times)$ to keep the samples in the hole, but seemed to preserve the complex better and yielded more particles with Npl4. Since the open state is a minor conformation (7\% of the total particles, Supplementary Fig. 1), the higher concentration of the CHAPSO dataset may have contributed to its discovery.

One critical finding in this study is the conformational changes of the ISS motifs in both ATPase rings in the translocating structures (Figs. If and 2a and Extended Data Fig. 5a,b). NMS-873 binds and locks the ISS motifs in the D2 ring to prevent the conformational changes (Fig. 4d-f), which further affects the pore loops and prevents substrate translocation. Targeting the ISS motif of p97, therefore, may serve as a potential strategy for the development of new inhibitors.

Multiple structures in the presence of NMS-873 at a substoichiometric concentration suggested that Npl4 may play a critical role in the initiation of translocation, by unfolding the ubiquitin chain and threading it into the central pore through a seesaw motion. The pore loops in the D1 and D2 rings catch the thread-in peptide, and the two rings are compressed to spiral conformations. Following sequential ATP hydrolysis and power-stroke motions circulating in both rings, the peptide in the pore is translocated downward. Npl4 might be unnecessary once the translocation starts.

Mutations in human $\mathrm{p} 97$ have been linked to $\mathrm{MSP}^{6,35}$. Structures of p97 in working states, therefore, provided crucial insights into the consequences of the mutations (Supplementary Table 3 and Supplementary Video 3). Furthermore, cryo-EM maps in various states allow a comprehensive understanding of substrate processing and allosteric inhibition of $\mathrm{p} 97$ and shed light on the mechanism of other type II AAA proteins.

\section{Online content}

Any methods, additional references, Nature Research reporting summaries, source data, extended data, supplementary information, acknowledgements, peer review information; details of author contributions and competing interests; and statements of data and code availability are available at https://doi.org/10.1038/ s41594-021-00617-2.

Received: 30 March 2021; Accepted: 4 June 2021; Published online: 14 July 2021

\section{References}

1. Bodnar, N. \& Rapoport, T. Toward an understanding of the Cdc48/p97 ATPase. F1000Res 6, 1318 (2017).

2. Erzberger, J. P. \& Berger, J. M. Evolutionary relationships and structural mechanisms of AAA+ proteins. Annu. Rev. Biophys. Biomol. Struct. 35, 93-114 (2006).

3. Rabouille, C., Levine, T. P., Peters, J.-M. \& Warren, G. An NSF-like ATPase, p97, and NSF mediate cisternal regrowth from mitotic Golgi fragments. Cell 82, 905-914 (1995).

4. van den Boom, J. \& Meyer, H. VCP/p97-mediated unfolding as a principle in protein homeostasis and signaling. Mol. Cell 69, 182-194 (2018).

5. Ye, Y. H., Tang, W. K. \& Xia, D. A mighty "protein extractor" of the cell: structure and function of the p97/CDC48 ATPase. Front. Mol. Biosci. 4, 39 (2017).

6. Tang, W. K. \& Xia, D. Mutations in the human AAA ${ }^{+}$chaperone p97 and related diseases. Front. Mol. Biosci. 3, 79 (2016).

7. Darwich, N. F. et al. Autosomal dominant VCP hypomorph mutation impairs disaggregation of PHF-tau. Science 370, eaay8826 (2020).

8. Huryn, D. M., Kornfilt, D. J. P. \& Wipf, P. p97: an emerging target for cancer, neurodegenerative diseases, and viral infections. J. Med. Chem. 63, 1892-1907 (2020).

9. Skrott, Z. et al. Alcohol-abuse drug disulfiram targets cancer via p97 segregase adaptor NPL4. Nature 552, 194-199 (2017).

10. Xia, D., Tang, W. K. \& Ye, Y. Structure and function of the AAA+ ATPase p97/Cdc48p. Gene 583, 64-77 (2016).

11. Huyton, T. et al. The crystal structure of murine p97/VCP at $3.6 \AA$. J. Struct. Biol. 144, 337-348 (2003).

12. Davies, J. M., Brunger, A. T. \& Weis, W. I. Improved structures of full-length p97, an AAA ATPase: implications for mechanisms of nucleotide-dependent conformational change. Structure 16, 715-726 (2008).

13. Banerjee, S. et al. 2.3 $\AA$ resolution cryo-EM structure of human p97 and mechanism of allosteric inhibition. Science 351, 871-875 (2016).

14. Zhang, X. et al. Structure of the AAA ATPase p97. Mol. Cell 6 , 1473-1484 (2000).

15. Ripstein, Z. A., Huang, R., Augustyniak, R., Kay, L. E. \& Rubinstein, J. L. Structure of a AAA plus unfoldase in the process of unfolding substrate. Elife 6, e25754 (2017).

16. Puchades, C. et al. Structure of the mitochondrial inner membrane AAA plus protease YME1 gives insight into substrate processing. Science 358, eaao0464 (2017)

17. Zhao, M. L. et al. Mechanistic insights into the recycling machine of the SNARE complex. Nature 518, 61-66 (2015).

18. Cooney, I. et al. Structure of the Cdc48 segregase in the act of unfolding an authentic substrate. Science 365, 502-505 (2019).

19. Twomey, E. C. et al. Substrate processing by the Cdc48 ATPase complex is initiated by ubiquitin unfolding. Science 365, eaax1033 (2019).

20. Alverez, C. et al. Allosteric indole amide inhibitors of p97: identification of a novel probe of the ubiquitin pathway. ACS Med. Chem. Lett. 7, 182-187 (2016)

21. Magnaghi, P. et al. Covalent and allosteric inhibitors of the ATPase VCP/p97 induce cancer cell death. Nat. Chem. Biol. 9, 548-556 (2013).

22. Zhang, T., Midshra, P., Hay, B. A., Chan, D. \& Guo, M. Valosin-containing protein $(\mathrm{VCP} / \mathrm{p} 97)$ inhibitors relieve Mitofusin-dependent mitochondrial defects due to VCP disease mutants. Elife 6, e17834 (2017).

23. Zhang, J. T. et al. Identification of NMS-873, an allosteric and specific p97 inhibitor, as a broad antiviral against both influenza A and B viruses. Eur. J. Pharm. Sci. 133, 86-94 (2019).

24. Bojkova, D. et al. Proteomics of SARS-CoV-2-infected host cells reveals therapy targets. Nature 583, 469-472 (2020).

25. Hao, Q. et al. A non-canonical role of the p97 complex in RIG-I antiviral signaling. EMBO J. 34, 2903-2920 (2015).

26. Pan, M. et al. Seesaw conformations of Npl4 in the human p97 complex and the inhibitory mechanism of a disulfiram derivative. Nat. Commun. 12, 121 (2021).

27. Blythe, E. E., Gates, S. N., Deshaies, R. J. \& Martin, A. Multisystem proteinopathy mutations in VCP/p97 increase NPLOC4.UFD1L binding and substrate processing. Structure 27, 1820-1829.e4 (2019).

28. Bodnar, N. O. \& Rapoport, T. A. Molecular mechanism of substrate processing by the Cdc48 ATPase complex. Cell 169, 722-735.e9 (2017).

29. Monroe, N., Han, H., Shen, P. S., Sundquist, W. I. \& Hill, C. P. Structural basis of protein translocation by the Vps4-Vtal AAA ATPase. Elife 6, e24487 (2017)

30. Yokom, A. L. et al. Spiral architecture of the Hsp104 disaggregase reveals the basis for polypeptide translocation. Nat. Struct. Mol. Biol. 23, 830-837 (2016). 
31. Hänzelmann, P. \& Schindelin, H. Structural basis of ATP hydrolysis and intersubunit signaling in the AAA+ ATPase p97. Structure 24, 127-139 (2016).

32. Blythe, E. E., Olson, K. C., Chau, V. \& Deshaies, R. J. Ubiquitin- and ATP-dependent unfoldase activity of P97/VCP.NPLOC4.UFD1L is enhanced by a mutation that causes multisystem proteinopathy. Proc. Natl Acad. Sci. USA 114, E4380-E4388 (2017).

33. Song, C., Wang, Q. \& Li, C.-C. H. ATPase activity of p97-valosin-containing protein (VCP). J. Biol. Chem. 278, 3648-3655 (2003).
34. Martin, A., Baker, T. A. \& Sauer, R. T. Rebuilt AAA+ motors reveal operating principles for ATP-fuelled machines. Nature 437, 1115-1120 (2005).

35. Meyer, H. \& Weihl, C. C. The VCP/p97 system at a glance: connecting cellular function to disease pathogenesis. J. Cell Sci. 127, 3877-3883 (2014).

Publisher's note Springer Nature remains neutral with regard to jurisdictional claims in published maps and institutional affiliations.

(C) The Author(s), under exclusive licence to Springer Nature America, Inc. 2021 


\section{Methods}

Protein expression and purification. Wild-type human p97, human p97 mutants, Npl4/Ufd1, His ${ }_{6}-\mathrm{Ub}^{\mathrm{G} 76 \mathrm{~V}}-\mathrm{Ub}^{\mathrm{G} 76 \mathrm{~V}}$-mEos3.2 (Ub-Eos), human UBA1, Ube25k and gp78RING-Ube2g2 were purified as previously described ${ }^{26,32,36}$. Briefly, all proteins were expressed in Escherichia coli BL21 (DE3) cells and purified through Ni-NTA resin at $4{ }^{\circ} \mathrm{C}$. All purified proteins were further buffer exchanged to the storage buffer containing $50 \mathrm{mM}$ HEPES, pH 8.0, $150 \mathrm{mM} \mathrm{NaCl}, 1 \mathrm{mM} \mathrm{MgCl}_{2}, 0.5 \mathrm{mM}$ tris(2-carboxyethyl)phosphine (TCEP) before snap freezing.

Preparation of polyubiquitinated Ub-Eos and polyubiquitin chain. For cryo-EM studies, His-tagged Ub-Eos was directly used for polyubiquitination. For substrate unfolding assays, Ub-Eos was first irradiated under light-emitting diode ultraviolet light (385-395 nm, uvBeast V2) for 1 hour at $4{ }^{\circ} \mathrm{C}$ to induce the photoconversion of $\mathrm{mEos} 3.2$, followed by polyubiquitination. The polyubiquitination reaction was performed as previously described ${ }^{32}$. Briefly, $20 \mu \mathrm{M}$ Ub-Eos was mixed with $1 \mu \mathrm{M}$ UBA1, $20 \mu \mathrm{M}$ gp78RING-Ube2g2 and $500 \mu \mathrm{M}$ ubiquitin in the ubiquitination buffer containing $20 \mathrm{mM}$ HEPES, pH 7.4, $150 \mathrm{mM} \mathrm{KCl,} 10 \mathrm{mM}$ ATP and $10 \mathrm{mM}$ $\mathrm{MgCl}_{2}$. After incubation at $37^{\circ} \mathrm{C}$ overnight, the mixture was incubated with $\mathrm{Ni}$-NTA resin to remove free ubiquitin chains. The elution was further separated using a Superdex 200 (GE Healthcare) size-exclusion column equilibrated in the storage buffer. Polyubiquitinated Ub-Eos ( $\mathrm{Ub}_{n}$-Eos) with longer ubiquitin chains (estimated over ten ubiquitin subunits) was collected and flash frozen for the substrate unfolding assay.

K48-linked polyubiquitin chains were assembled by mixing $1 \mu \mathrm{M}$ UBA1, $10 \mu \mathrm{M}$ Ube25k and $1 \mathrm{mM}$ ubiquitin in the ubiquitination buffer at $37^{\circ} \mathrm{C}$ for 4 hours. The reaction mixture was then diluted tenfold with $50 \mathrm{mM} \mathrm{NaOAc}$, $\mathrm{pH} 4.5$, and separated by a Mono S cation exchange column (GE Healthcare). The corresponding peak for hexa-ubiquitin chain $\left(\mathrm{Ub}_{6}\right)$ was collected and further purified with a Superdex 75 (GE Healthcare) size-exclusion column equilibrated in the storage buffer.

\section{Assembly of substrate-engaged p97 complex. p97-Npl4/Ufd1 complex was assembled as previously described ${ }^{26}$. A p97 mutant bearing A232E and E578Q mutations was used to decrease the heterogeneity of $\mathrm{N}$ domains and reduce the unfolding activity of the complex. Twofold molar excess of $\mathrm{Ub}_{6}$ or $\mathrm{Ub}_{n}$-Eos was added to the $\mathrm{p} 97-\mathrm{Npl} 4 / \mathrm{Ufd} 1$ complex, followed by gel filtration using a Superose 6 column (GE Healthcare) equilibrated in the storage buffer (Extended Data Fig. $1 \mathrm{~b}, \mathrm{c})$. The assembled substrate-engaged p97 complex was flash frozen in liquid nitrogen for structural studies. No nucleotide was supplemented during the assembly.}

Specimen preparation for single-particle cryo-EM. All samples for cryo-EM were prepared as previously described ${ }^{26}$, with the following modifications. Either FOM or CHAPSO was used to relieve the preferred orientations, instead of IGEPAL CA-630. The complex was concentrated to $\sim 2.5 \mathrm{mg} \mathrm{ml}^{-1}$ followed by incubation with $5 \mathrm{mM}$ ATP at room temperature for 5 minutes before adding FOM (final concentration $0.01 \% \mathrm{v} / \mathrm{v}$ ). Glow-discharged Quantifoil Cu 1.2/1.3 grids were used for FOM-supplemented samples. Alternatively, the samples were concentrated to $\sim 20 \mathrm{mg} \mathrm{ml}^{-1}$ followed by incubation with $5 \mathrm{mM}$ ATP at room temperature for 5 minutes before adding CHAPSO (final concentration $4 \mathrm{mM}$ ). Non-glow-discharged Quantifoil $\mathrm{Cu} 1.2 / 1.3$ grids were used for CHAPSO-supplemented samples. In both cases, 3.5- $\mu$ l samples were applied to the grid and blotted for 1 second using standard Vitrobot filter paper (Ted Pella, catalog no. 47000-100) before plunge freezing in liquid ethane. For substrate-engaged p97-Npl4/Ufd 1 complex in the presence of NMS-873, besides the aforementioned procedures, NMS-873 was supplemented right after the incubation with ATP at either $10 \mu \mathrm{M}$ or $80 \mu \mathrm{M}$ final concentration. The samples were incubated for another $30 \mathrm{~min}$ at room temperature before adding the detergents and plunge freezing.

Data collection for single-particle cryo-EM. Data collection was performed either in the Advanced Electron Microscopy Facility at the University of Chicago or the National Cryo-Electron Microscopy Facility at the National Cancer Institute. All datasets were acquired as movie stacks with a Titan Krios electron microscope operating at $300 \mathrm{kV}$, equipped with either a Gatan K2 Summit or K3 direct detector camera. A single stack typically consists of 40 frames with a total exposure around 50 electrons $/ \AA^{2}$. The defocus range was set at -1.0 to $-2.5 \mu \mathrm{m}$. See Tables $1-3$ and Supplementary Tables 1 and 2 for details.

Image processing. Movie stacks were subjected to beam-induced motion correction using MotionCor2 (ref. ${ }^{37}$ ). CTF parameters for each micrograph were determined using CTFFIND4 (ref. ${ }^{38}$ ). Particle picking, two- and three-dimensional classifications, three-dimensional refinement and local resolution estimation were performed in RELION-3 (ref. ${ }^{39}$ ). Particle picking was performed by manually choosing $\sim 2,000$ particles and generating templates through reference-free two-dimensional (2D) classification, followed by automatic template-based picking. False-positive particles or particles classified in poorly defined classes were discarded after 2D classification. The initial 3D classification was performed on a binned dataset with the previously reported p 97 structures as the reference model $^{26}$. The detailed data processing flows are shown in Supplementary Figs. 1-6.
To make sure that the 3D classification did not miss any major conformations, additional runs were performed with different number of classes (ranges from 3 to 8 ) and different regularization parameters (ranges from 2 to 6). Since we did not observe a dependence on the regularization parameter, the results from the default value $(T=4)$ are shown in the figures. Data processing statistics are summarized in Tables 1-3 and Supplementary Tables 1 and 2. Reported resolutions are based on Fourier shell correlation (FSC) using the $\mathrm{FSC}=0.143$ criterion.

Model building, refinement and validation. Model building was based on the existing cryo-EM structures of human p97 (ref. ${ }^{26}$ ) (PDB 7JY5). The models of individual domains (N, D1 and D2) were first docked into the cryo-EM maps as rigid bodies using UCSF Chimera ${ }^{40}$ followed by further adjustment using $\operatorname{Coot}^{41}$. For well-resolved regions, sharpened maps were used and individual residues were manually adjusted and fit into the density. For flexible regions, unsharpened maps were used and individual secondary structure elements or subdomains were fit into the density as rigid bodies. A short $\beta$-strand was automatically built into the density corresponding to the translocating peptide using Coot, followed by manual extension on either end and real-space refinement, until the all the density was covered. The final models were subjected to global refinement and minimization in real space using the real-space refinement module in Phenix ${ }^{42}$. Model validation was performed using the comprehensive validation tool in Phenix. The statistics of model refinement are shown in Tables 1-3 and Supplementary Table 1. Structure figures were made using ChimeraX v.0.9 (ref. ${ }^{43}$ ) and PyMOL v.2.4.0 (The PyMOL Molecular Graphics System, Schrödinger).

Substrate unfolding assay. Substrate unfolding assay was performed as previously described $^{26,32}$. Briefly, $20 \mathrm{nM}$ photoconverted polyubiquitinated Ub-Eos was mixed with $400 \mathrm{nM}$ p 97 or p 97 mutants and $500 \mathrm{nM}$ Npl4/Ufd1 in the assay buffer containing $50 \mathrm{mM}$ Tris $\mathrm{pH} 7.4,5 \mathrm{mM} \mathrm{KCl}, 20 \mathrm{mM} \mathrm{MgCl}_{2}, 1 \mathrm{mM}$ EDTA, $0.5 \mathrm{mM}$ TCEP and $0.01 \%$ Triton. Proteins were preincubated in a 96 -well plate (Costar, catalog no. 3694) for 10 minutes at $37^{\circ} \mathrm{C}$ before initiating the reaction by supplementing the ATP regeneration mixture ( $5 \mathrm{mM}$ ATP, $30 \mathrm{mM}$ creatine phosphate and $50 \mu \mathrm{g} \mathrm{ml}^{-1}$ creatine phosphokinase). Fluorescence signal was monitored using a TECAN safire 2 plate reader at $540 \mathrm{~nm}$ excitation and $580 \mathrm{~nm}$ emission wavelengths with 30 -second intervals for 60 minutes. Each reaction condition was repeated three times. Background fluorescence was measured by mixing the same amount of substrate with $6 \mathrm{M}$ guanidine- $\mathrm{HCl}$ and was subtracted from the average of the experimental groups. Normalized fluorescence was plotted using GraphPad Prism v.8.4.2.

Reporting Summary. Further information on research design is available in the Nature Research Reporting Summary linked to this article.

\section{Data availability}

Cryo-EM maps have been deposited in the Electron Microscopy Data Bank (EMDB, www.ebi.ac.uk/pdbe/emdb/) under the accession codes

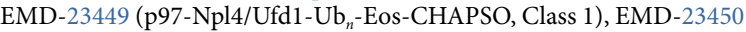

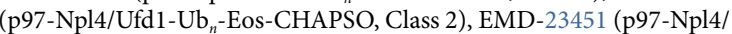
Ufd1-Ub ${ }_{n}$-Eos-CHAPSO, Class 3), EMD-23446 (p97-Npl4/Ufd1-Ub ${ }_{n}$-Eos-FOM, Class 1), EMD-23447 (p97-Npl4/Ufd1-Ub ${ }_{n}$-Eos-FOM, Class 2), EMD-23448 (p97-Npl4/Ufd1-Ub ${ }_{n}$-Eos-FOM, Class 3), EMD-23443 (p97-Npl4/Ufd1-Ub 6 -FOM, Class 1), EMD-23444 (p97-Npl4/Ufd1-Ub - FOM, Class 2), EMD-23445 (p97-Npl4/Ufd1-Ub 6 -FOM, Class 3), EMD-23442 (p97-Npl4/Ufd1-Ub 6 -NMS873-FOM), EMD-23452 (p97-Npl4/Ufd1-Ub -Eos-NMS-873-CHAPSO), EMD-

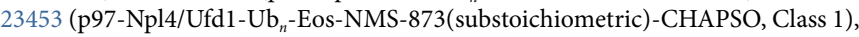
EMD-23454 (p97-Npl4/Ufd1-Ub -Eos-NMS-873(substoichiometric)-CHAPSO,

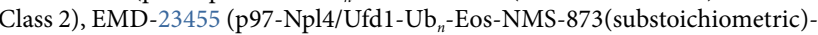

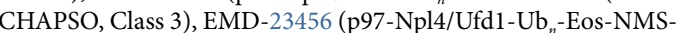
873(substoichiometric)-CHAPSO, Class 4), EMD-23457 (p97-Npl4/ Ufd1-Ub ${ }_{n}$-Eos-NMS-873(substoichiometric)-CHAPSO, Class 5), EMD-23458 (p97-Npl4/Ufd1-Ub - -Eos-NMS-873(substoichiometric)-CHAPSO, Class 6). The atomic models have been deposited in the Protein Data Bank under the

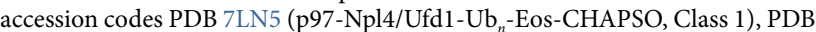

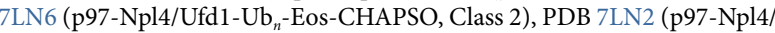

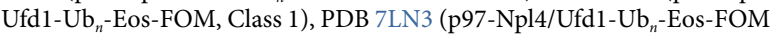

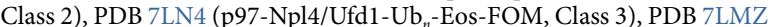

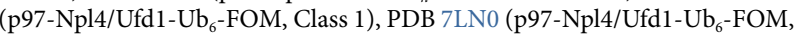
Class 2), PDB 7LN1 (p97-Npl4/Ufd1-Ub - -FOM, Class 3), PDB 7LMY (p97-Npl4/ Ufd1-Ub $\left.{ }_{6}-\mathrm{NMS}-873-\mathrm{FOM}\right)$. Source data are provided with this paper.

\section{References}

36. Pickart, C. M. \& Raasi, S. Controlled synthesis of polyubiquitin chains. Methods Enzymol. 399, 21-36 (2005).

37. Zheng, S. Q. et al. MotionCor2: anisotropic correction of beam-induced motion for improved cryo-electron microscopy. Nat. Methods 14, 331-332 (2017)

38. Mindell, J. A. \& Grigorieff, N. Accurate determination of local defocus and specimen tilt in electron microscopy. J. Struct. Biol. 142, 334-347 (2003). 
39. Zivanov, J. et al. New tools for automated high-resolution cryo-EM structure determination in RELION-3. Elife 7, e42166 (2018).

40. Pettersen, E. F. et al. UCSF Chimera: a visualization system for exploratory research and analysis. J. Comput. Chem. 25, 1605-1612 (2004).

41. Emsley, P. \& Cowtan, K. Coot: model-building tools for molecular graphics. Acta Crystallogr. Sect. D Struct. Biol. 60, 2126-2132 (2004).

42. Adams, P. D. et al. PHENIX: a comprehensive Python-based system for macromolecular structure solution. Acta Crystallogr. Sect. D Struct. Biol. 66, 213-221 (2010).

43. Pettersen, E. F. et al. UCSF ChimeraX: structure visualization for researchers, educators, and developers. Protein Sci. 30, 70-82 (2021).

\section{Acknowledgements}

We thank staff at the National Cryo-Electron Microscopy Facility at the Frederick National Laboratory and the Advanced Electron Microscopy Facility at the University of Chicago for their help in cryo-EM data collection. Funding for this work was, in part, provided by the Catalyst Award from the Chicago Biomedical Consortium. This work was supported by Chicago Biomedical Consortium Catalyst Award C-086 (to M.Z.). We thank the National Key R\&D Program of China (no. 2017YFA0505200) and NSFC $(91753205,81621002,21621003)$ for financial support. This research was, in part, supported by the National Cancer Institute's National Cryo-EM Facility at the Frederick National Laboratory for Cancer Research under contract HSSN261200800001E.

\section{Author contributions}

M.P., M.Z. and L.L. designed all of the experiments and interpreted the results. M.P., Y.Y., H.A., Q.Z. and Y.X. cloned, expressed and purified all of the complexes and carried out related biochemical characterizations. M.P., Y.Y. and M.Z. performed cryo-EM data collection and processing. M.Z., M.P. and L.L. wrote the paper. M.Z., L.L. and M.P. supervised the project.

\section{Competing interests}

The authors declare no competing interests.

\section{Additional information}

Extended data is available for this paper at https://doi.org/10.1038/s41594-021-00617-2.

Supplementary information The online version contains supplementary material available at https://doi.org/10.1038/s41594-021-00617-2.

Correspondence and requests for materials should be addressed to M.P., L.L. or M.Z.

Peer review information Nature Structural \& Molecular Biology thanks Edward Twomey for their contribution to the peer review of this work. Peer reviewer reports are available. Anke Sparmann was the primary editor on this article and managed its editorial process and peer review in collaboration with the rest of the editorial team.

Reprints and permissions information is available at www.nature.com/reprints. 
a

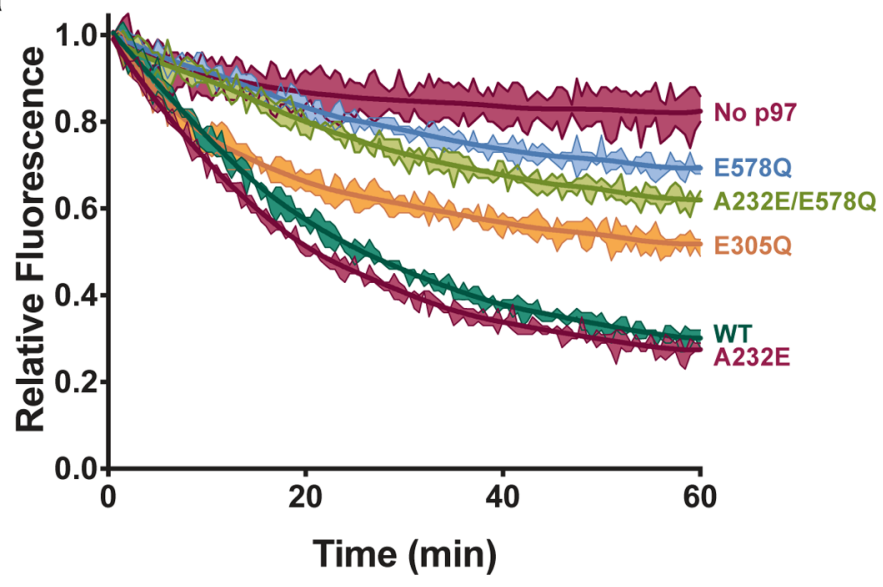

b

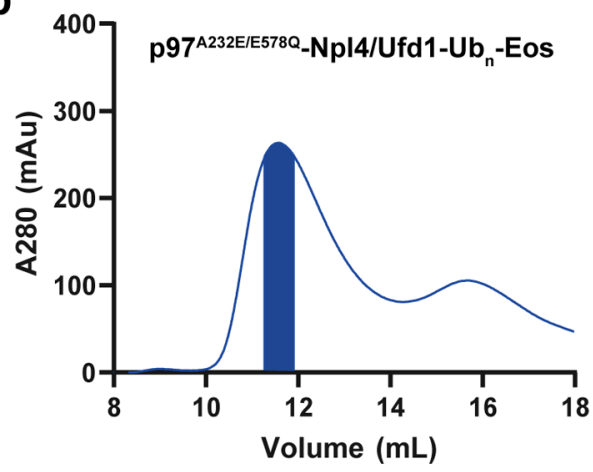

C

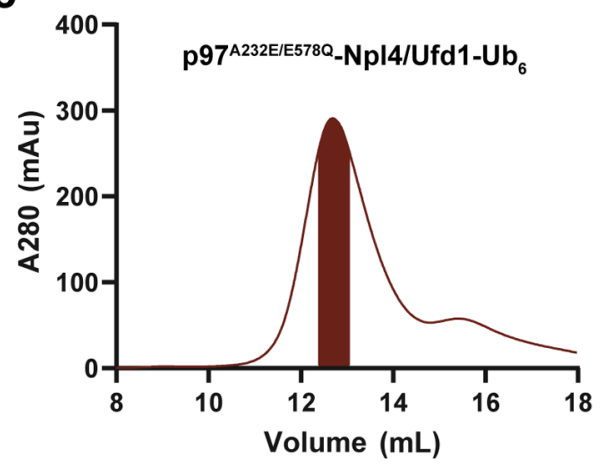

d

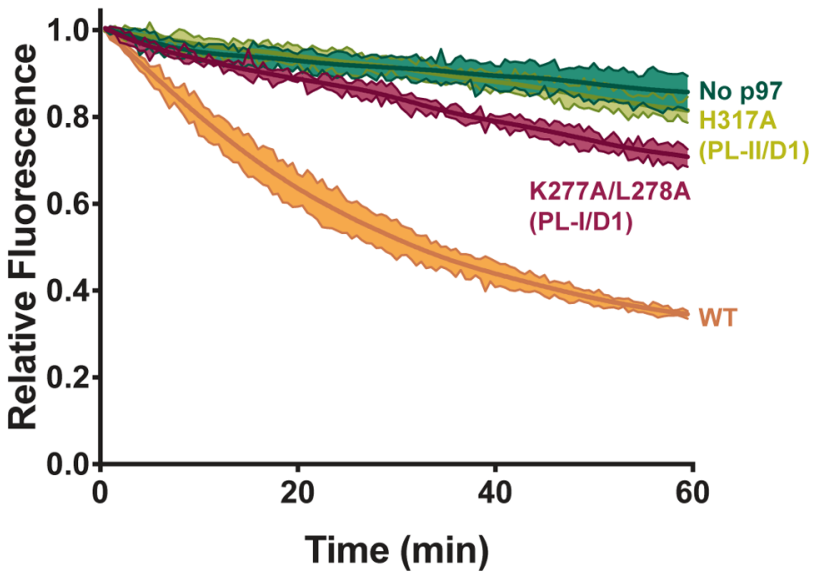

e

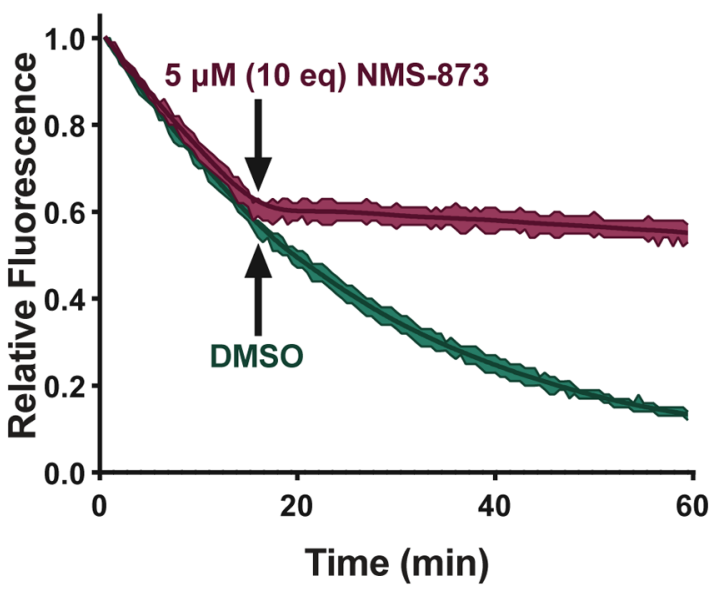

Extended Data Fig. 1 | Substrate unfolding activity of various p97 mutants and the purification of cofactor- and substrate-engaged p97 complexes. a, Substrate unfolding assay for wild-type (WT) p97 and various mutations. E305Q and E578Q are the two Walker B mutations in the D1 and D2 domains, respectively. A232E is a disease mutation found in multisystem proteinopathy (MSP) ${ }^{27,32}$. b. Gel filtration chromatogram and SDS-PAGE gel of the p97 ${ }^{A 232 E /}$

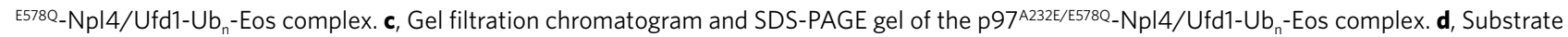
unfolding assay for the pore loop mutants in the D1 domain of p97. e, Substrate unfolding assay for wild-type p97. NMS-873 or DMSO was introduced 15 minutes after the reaction. The curves in panels $\mathbf{a}, \mathbf{d}$, and $\mathbf{e}$ are presented as mean values \pm SD from triplicate experiments. 
a
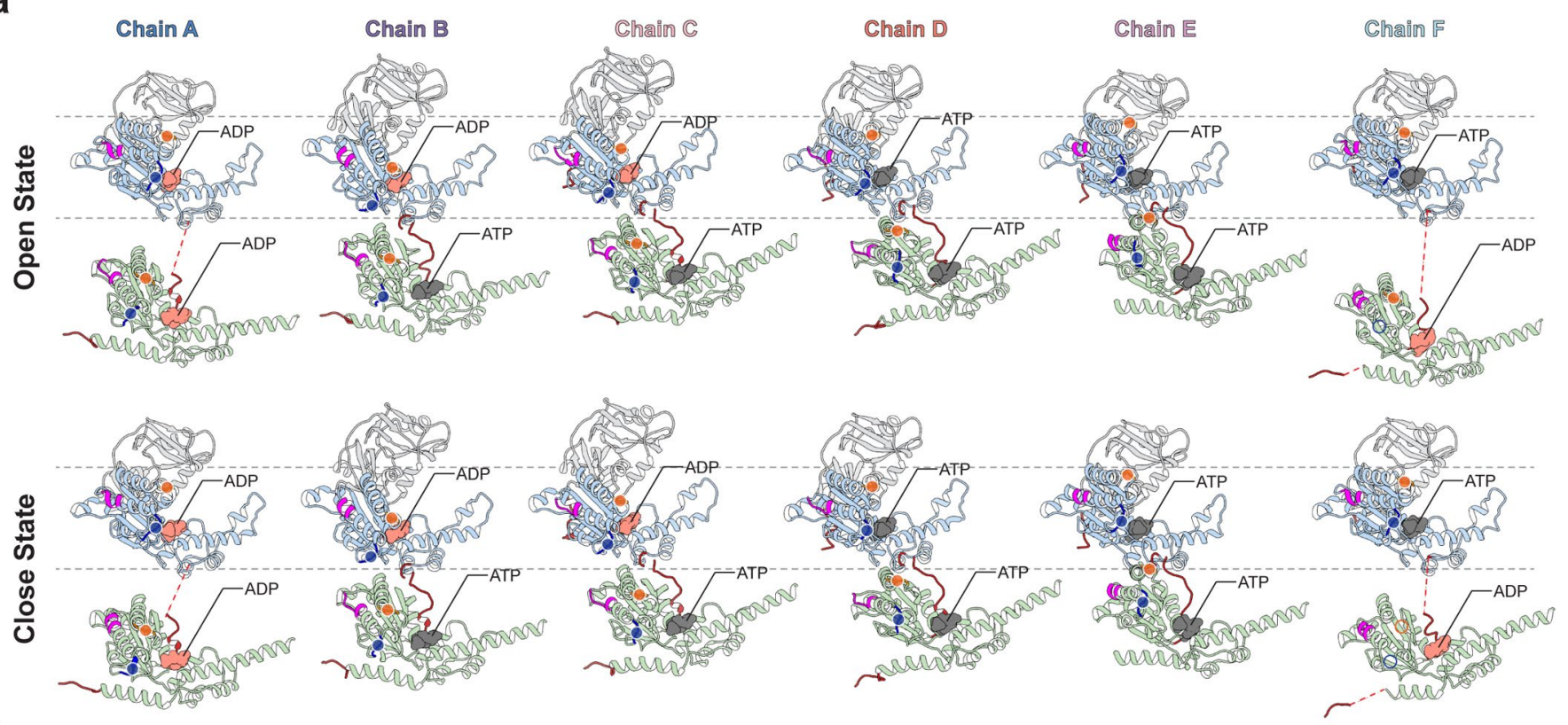

b

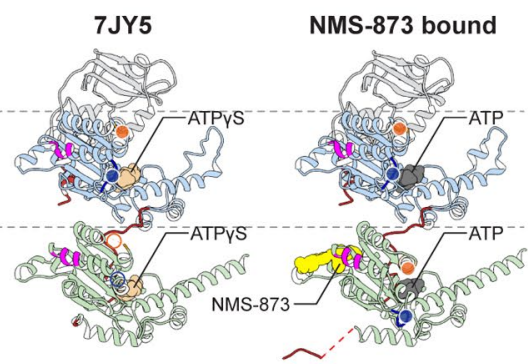

C
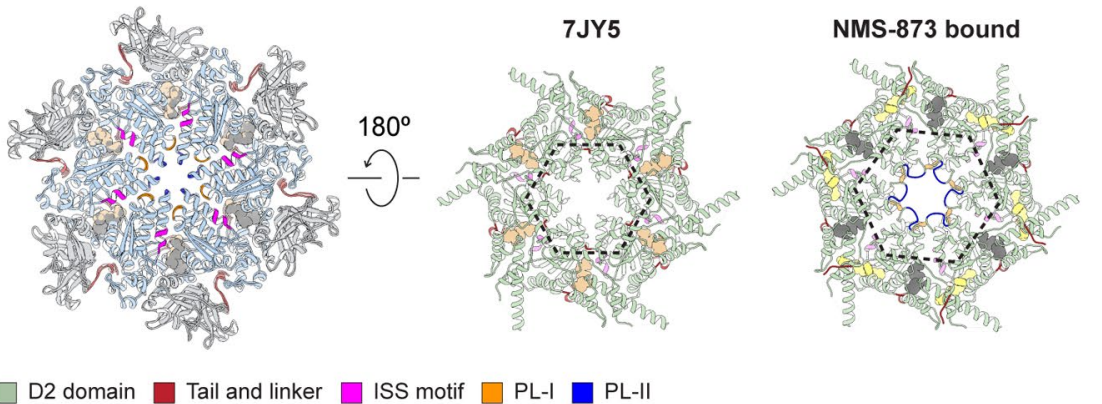

d
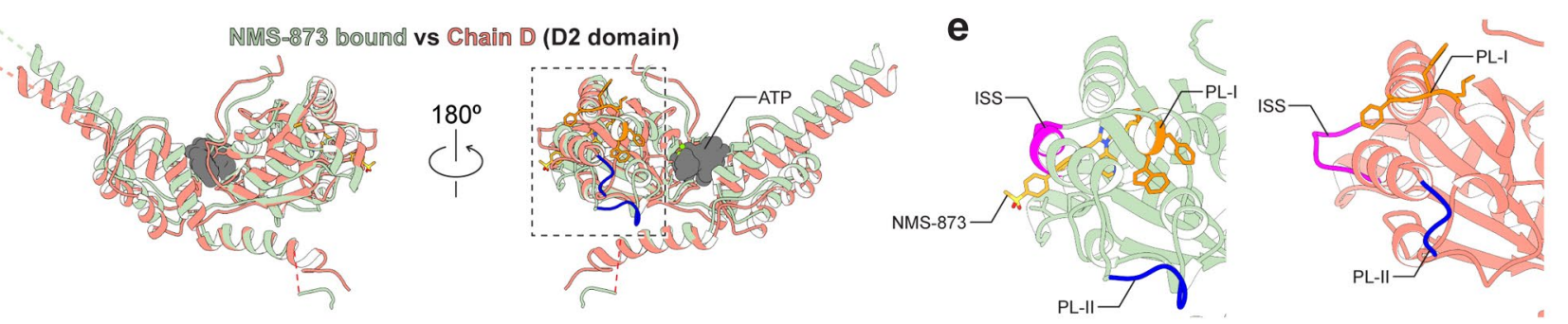

$\mathbf{f}$
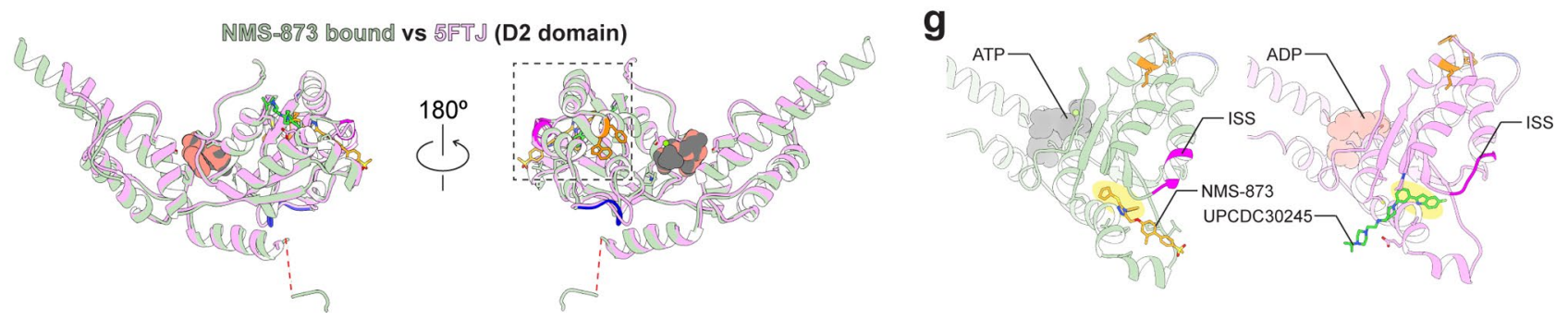

Extended Data Fig. 2 | Comparison of various p97 structures. a, Unrolling of the open (top row) and closed (bottom row) states of p97. The lateral and vertical movement of D2 domains can be visualized. The positions of pore loops are marked with solid dots. Unresolved pore loops are marked by open circles. b, Unrolling of the ATP $\gamma$ S-bound nontranslocating structure (PDB ID: 7JY5) and NMS-873-bound structure. Only one chain of each structure is shown since both structures are sixfold symmetric. c, A superimposition of 7JY5 and NMS-873-bound structures. Left: a top view of the N and D1 domains; Right: a bottom view of the D2 ring. The opening of the D2 ring is marked by a dotted hexagon. Panels $\mathbf{a}, \mathbf{b}$, and $\mathbf{c}$ share the same color code. d, A comparison of the D2 domain in the NMS-873-bound structure and that in chain D of the open state. The superimposition is based on the $\alpha / \beta$ subdomain. e, Magnified views of the dotted box in panel d, showing the conformations of the PL-I, PL-II, and ISS motifs. $\mathbf{f}$, A comparison of the D2 domain in NMS-873- and UPCDC30245 (PDB ID: 5FTJ)-bound structures. The superimposition is based on the $\alpha / \beta$ subdomain. $\mathbf{g}$, Magnified views of the dotted box in panel $\mathbf{f}$, showing the binding sites of the compounds and the conformations of the ISS motif. 
a

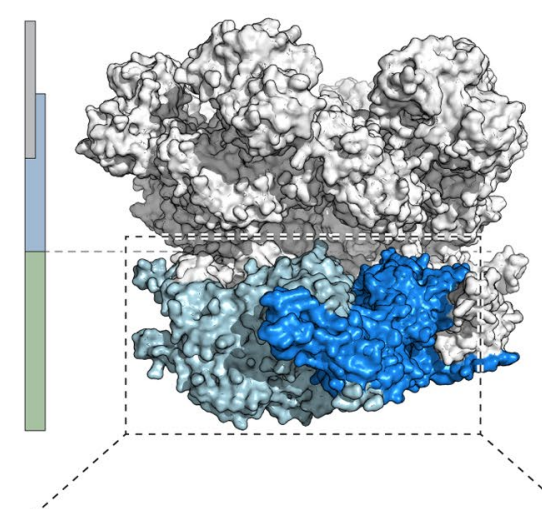

b

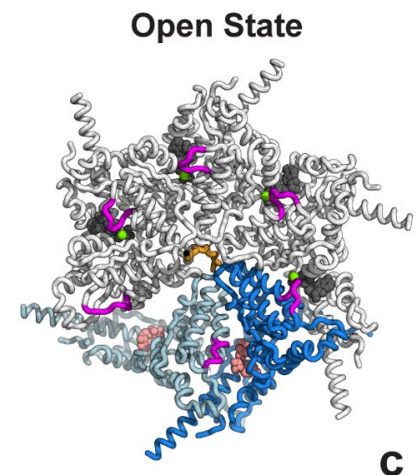

Close State

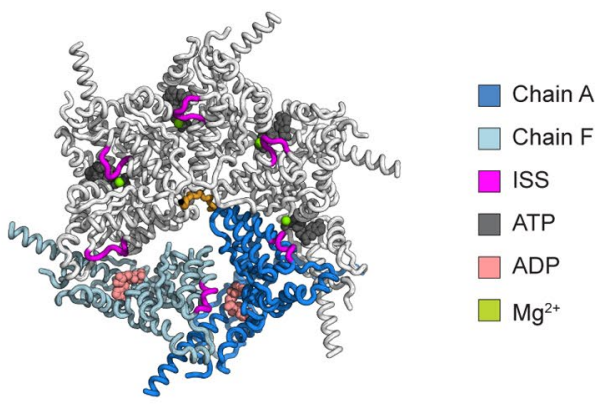

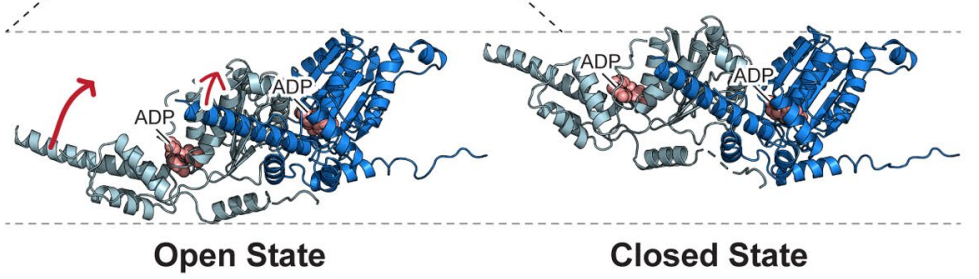

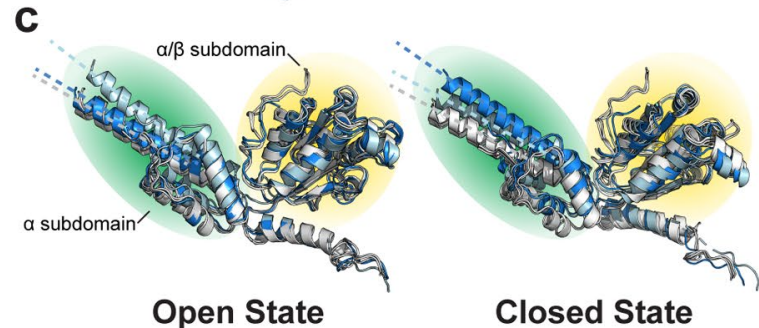

d

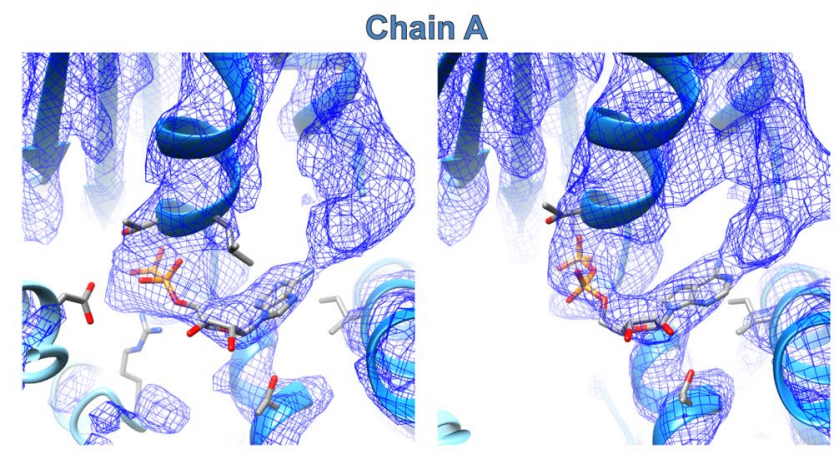

Chaำ $\mathbb{C}$

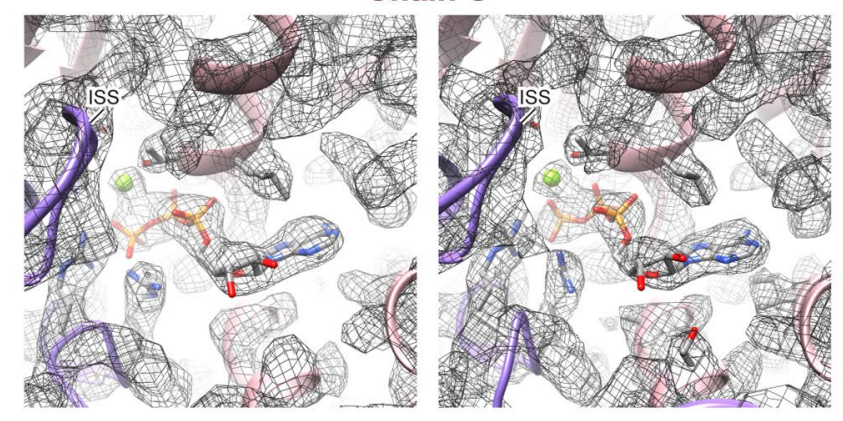

Chån ⿷
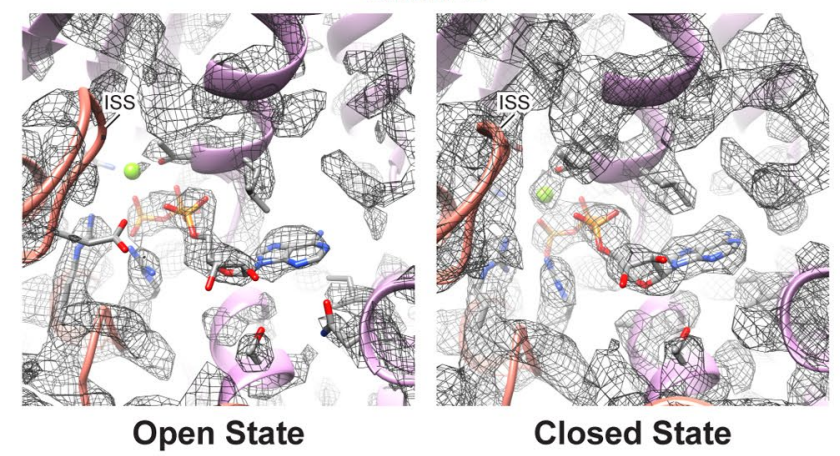

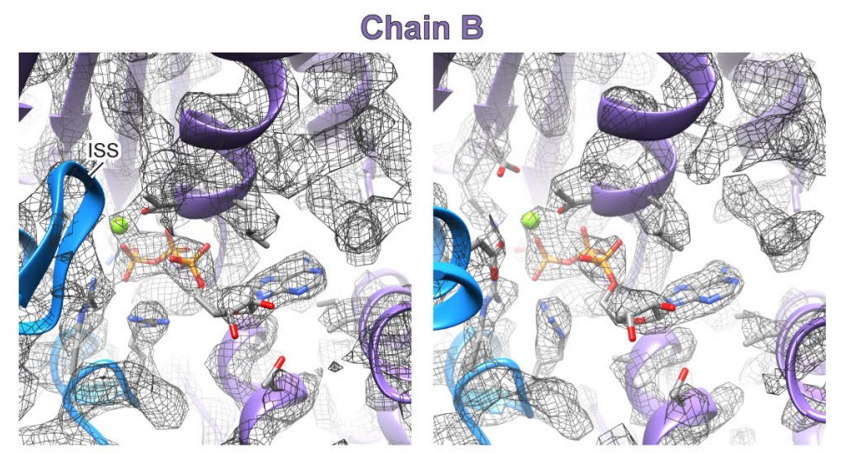

Chain D

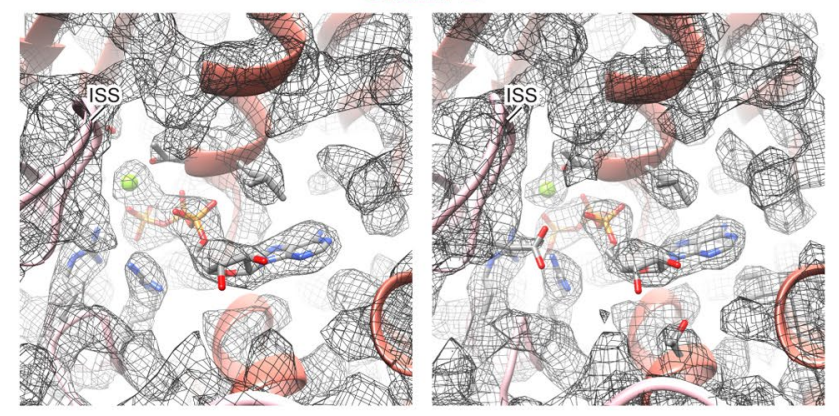

Chaำ F

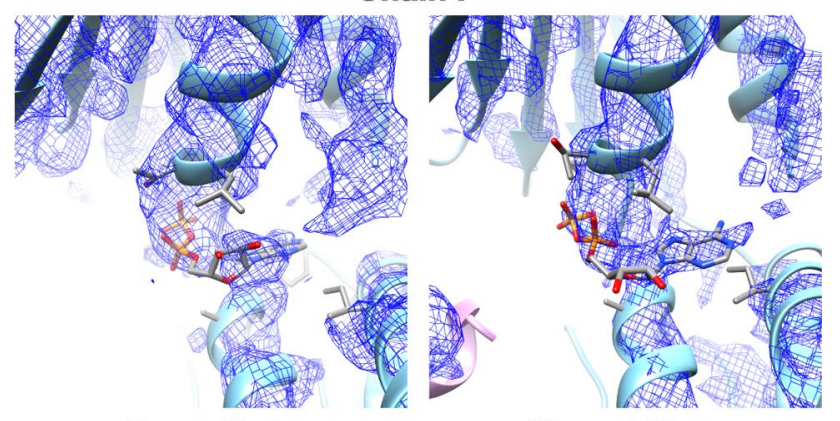

Open State

Closed State

Extended Data Fig. 3 | See next page for caption. 
Extended Data Fig. 3 | The power stroke motion and the nucleotide binding sites of the D2 domains. a, A superimposition of the open and closed state structures, with two D2 domains (chain A and chain F) magnified. The relative positions of the D2 domains in chain A and chain F can be visualized. $\mathbf{b}$, Top views of the D2 ring in the open and closed states, highlighting chain A, chain F, and the ISS motifs. c, Superimpositions of all D2 domains in the open and closed states based on the $\alpha / \beta$ subdomain. The directions of $\alpha 7$ helices are highlighted by dotted lines for comparison (dark blue, chain $A$; light blue, chain F; and gray, other chains). d, Individual nucleotide binding sites of the D2 domains in the open and closed states. Blue mesh: unsharpened map at contour level 0.02; Gray mesh: sharpened map at contour level 0.05 . 
a

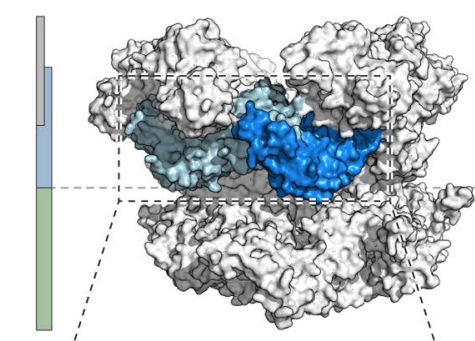

b

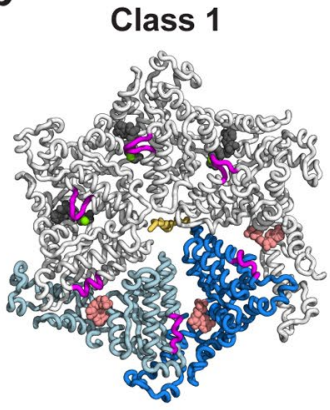

Class 2

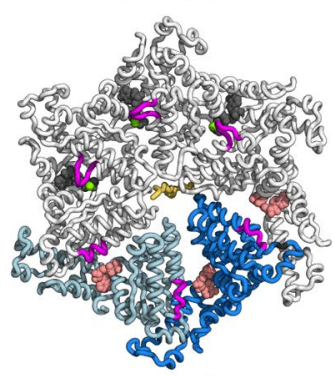

Class 3

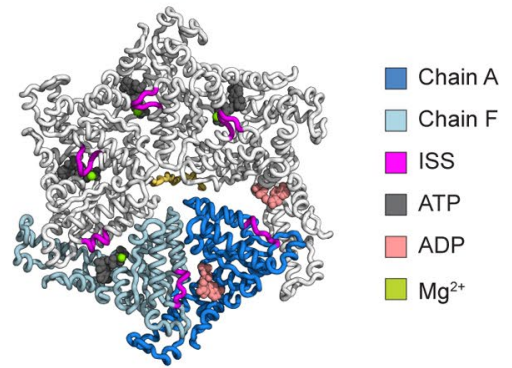

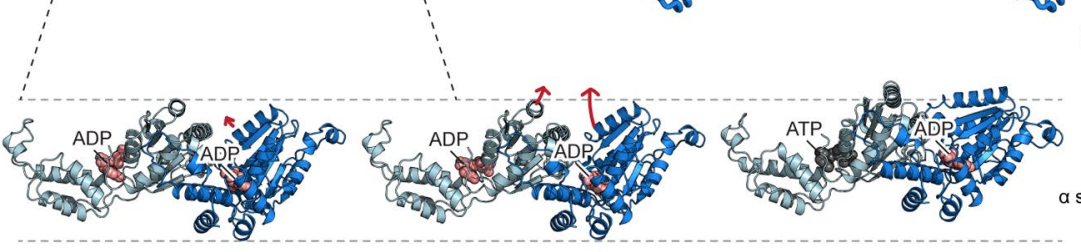

Class 1

Class 2

Class 3

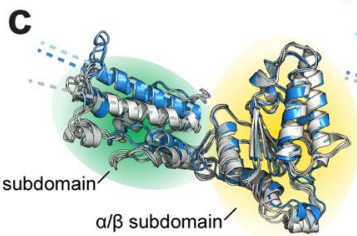

Class 1

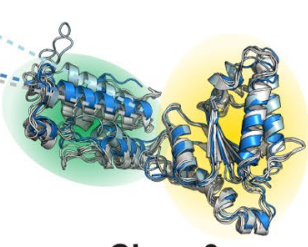

Class 3

d
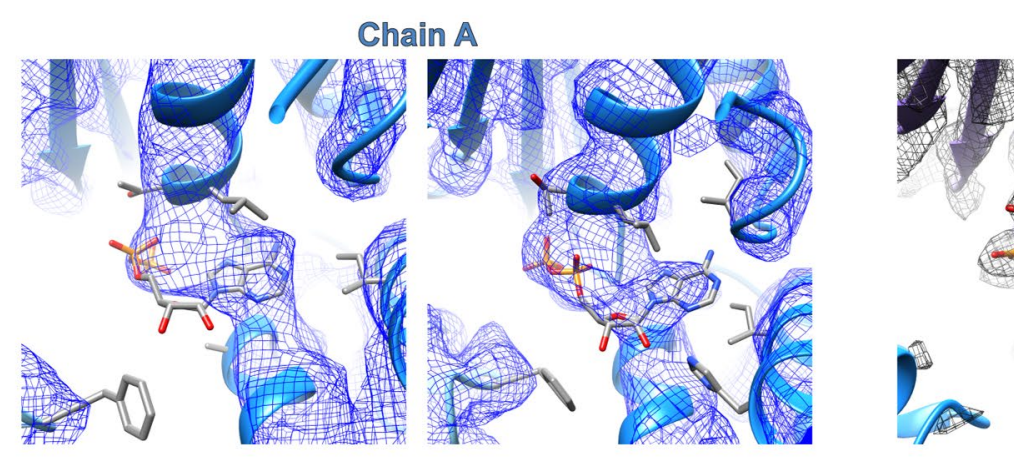

Chain B
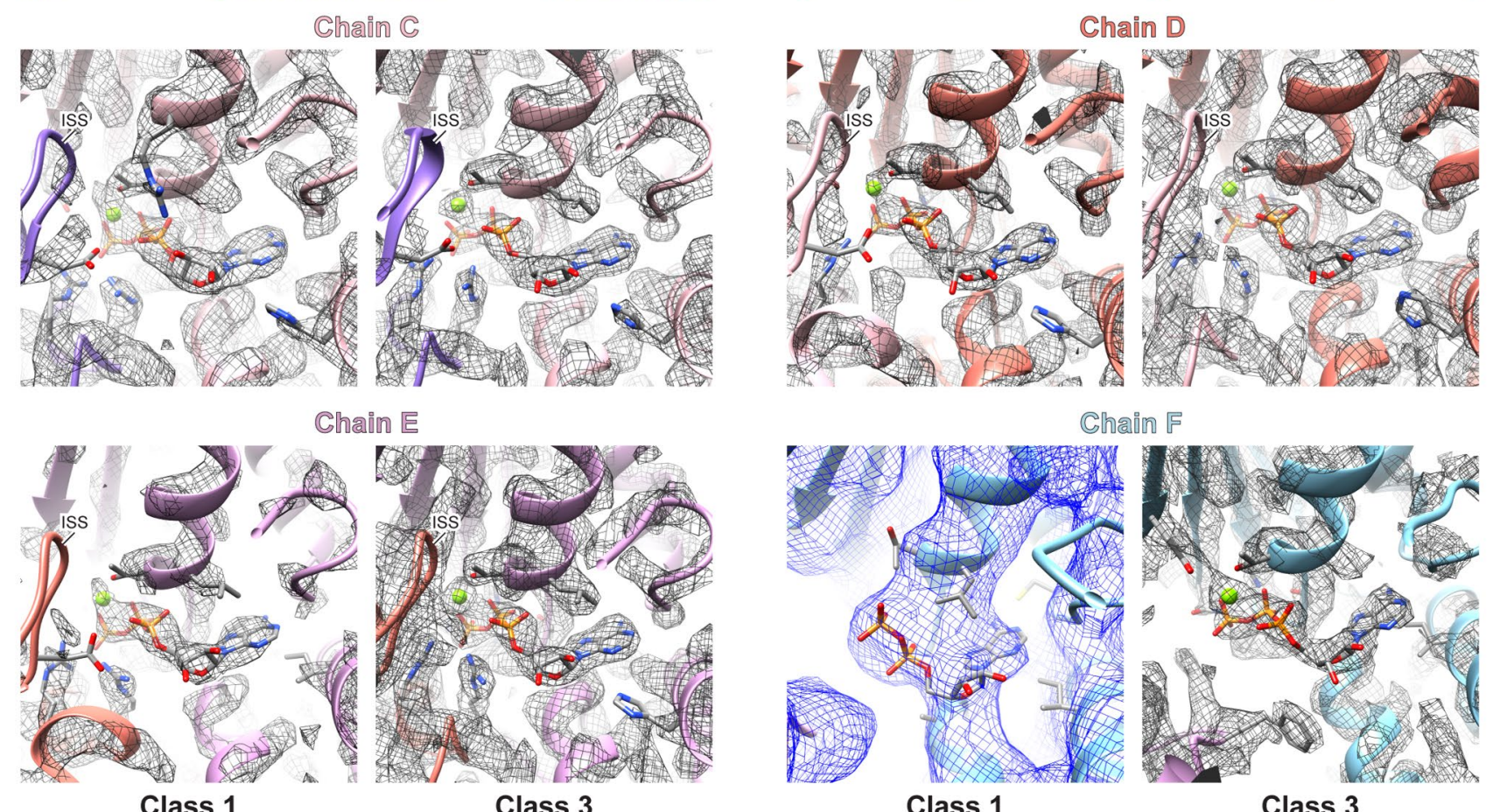

Chain E

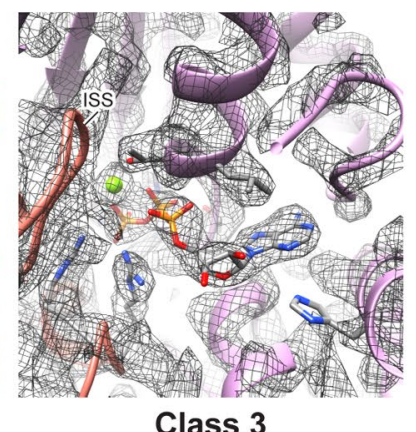

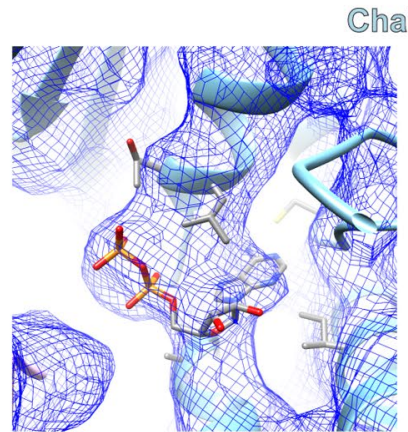

Class 1

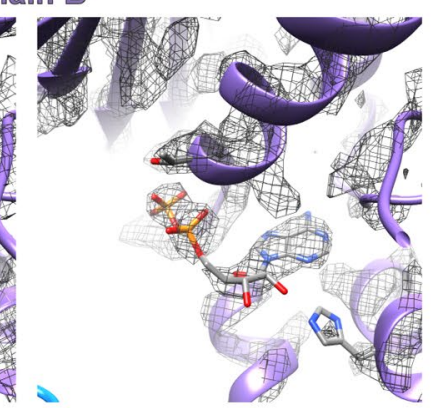

Chain D

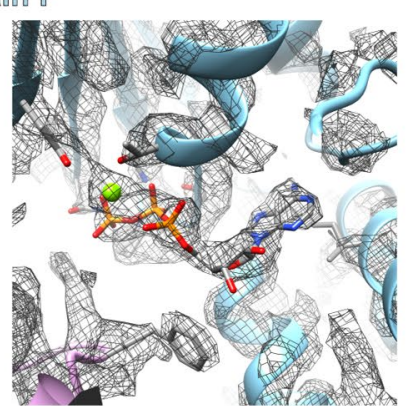

Class 3

Extended Data Fig. 4 | The power stroke motion and the nucleotide binding sites of the D1 domains. a, A superimposition of three structures (classes 1, 2, and 3) from the p97-Npl4/Ufd1-Ub -Eos-FOM dataset, with two D1 domains (chain A and chain F) magnified. The relative positions of the D1 domains in chain A and chain F can be visualized. b, Top views of the D1 ring in the three classes, highlighting chain A, chain F, and the ISS motifs. c, Superimpositions of all D1 domains in class 1 and class 3 based on the $\alpha / \beta$ subdomain. The directions of $\alpha 7$ helices are highlighted by dotted lines for comparison (dark blue, chain A; light blue, chain F; and gray, other chains). d, Individual nucleotide binding sites of the D1 domains in class 1 and class 3. Blue mesh: Unsharpened map at contour level 0.015; Gray mesh: sharpened map at contour level 0.04. 
a

b
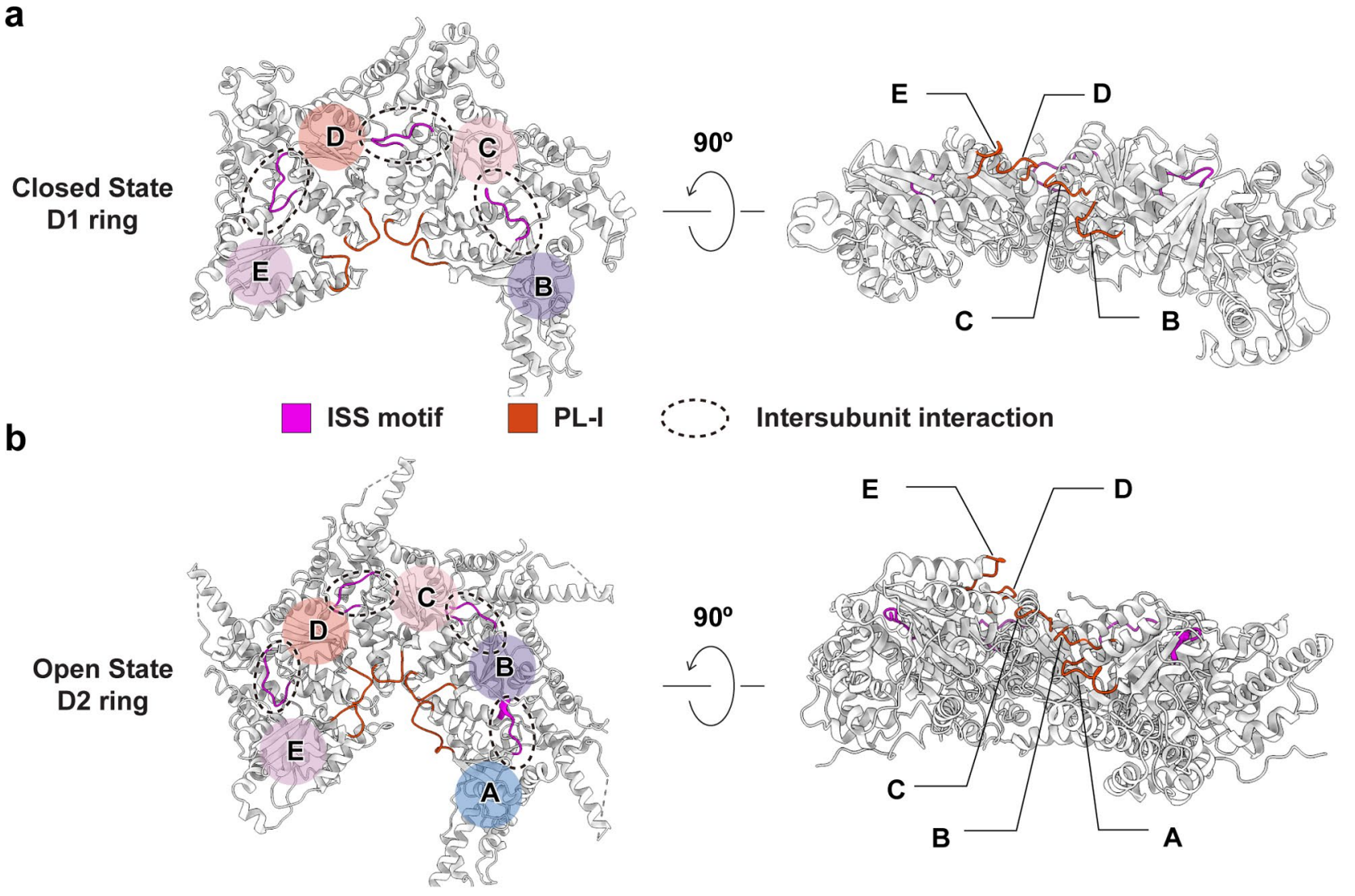

C
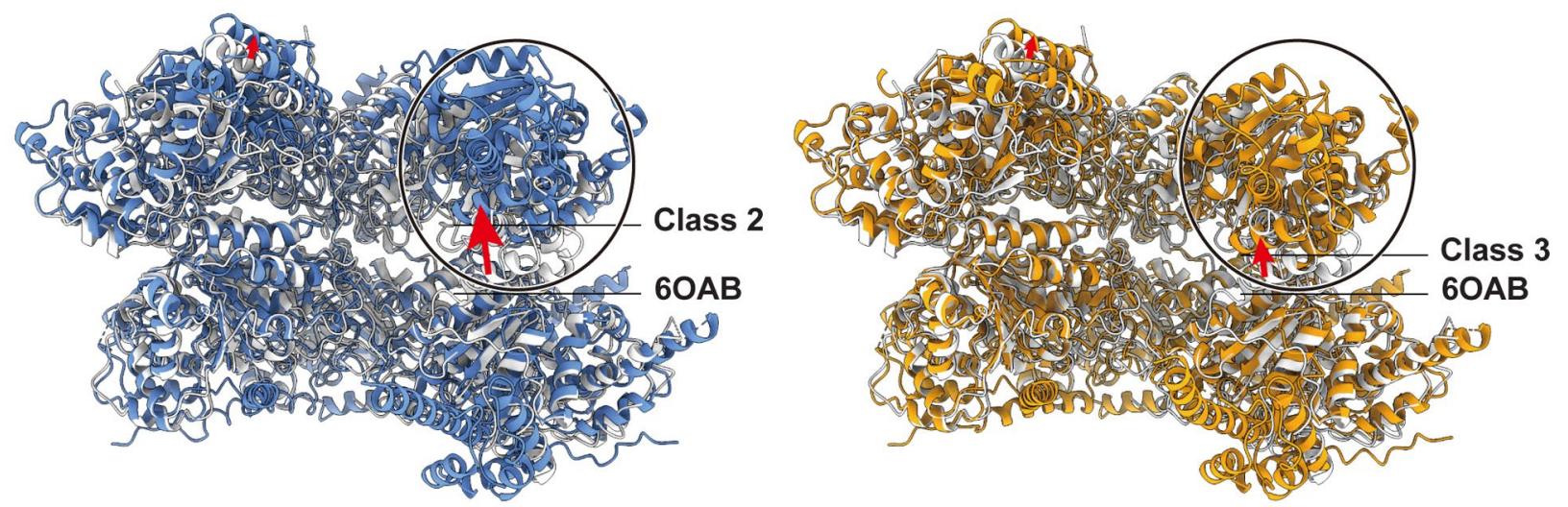

Cdc48 (ADP-BeFx) $\square$ Class $2 \square$ Class $3 \bigcirc$ D1 domain of Chain A $\because:$ Flexible domains

d
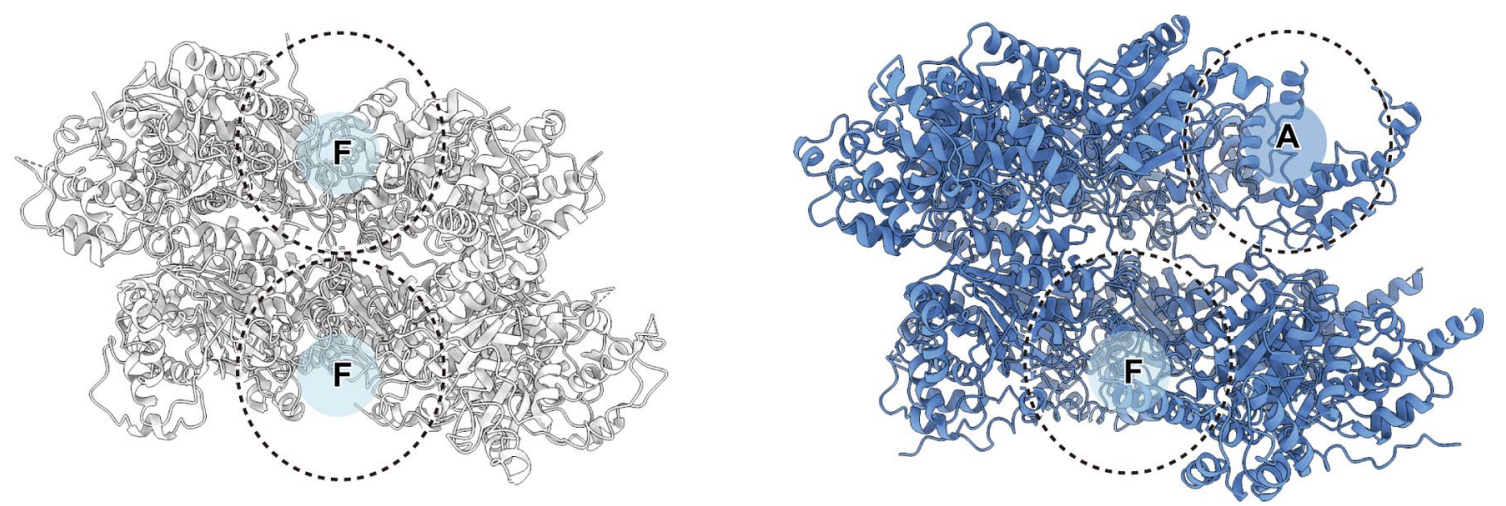

Extended Data Fig. 5 | See next page for caption. 
Extended Data Fig. 5 | Comparisons of human p97 and yeast Cdc48 structures. a-b, Illustrations showing the arrangement of chains B-E in the D1 ring of the closed state (a) and chains A-E in the D2 ring of the open state (b). The labels of chains share the same color code as in Fig. 1e. c, Comparisons of

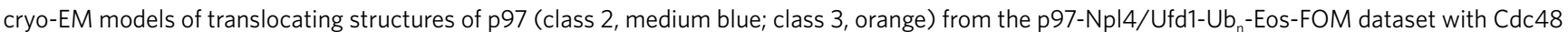
(ADP-BeFx, PDB ID: 6OAB, light grey). The models were aligned based on the D2 ring. The relative shifts of the D1 domain in chain A are noted by red arrows. d, Illustrations showing the model of Cdc48 (ADP-BeFx, PDB ID: 6OAB, light grey, left panel) and the translocating structure of p97 (class 2 , medium blue, right panel) without the flexible domains. The entire chain $\mathrm{F}$ in $\mathrm{Cdc} 48$ (ADP-BeFx) was flexible according to the previous publication ${ }^{19}$.

The D2 domain in chain F and D1 domain in chain A were flexible in this study. Both (positioned in dotted circles) were hid for comparison. Note that the staircases formed by D1 and D2 rings are in register for $\mathrm{Cdc} 48$ and out of register for $\mathrm{p} 97$. 


\section{Reporting Summary}

Nature Research wishes to improve the reproducibility of the work that we publish. This form provides structure for consistency and transparency in reporting. For further information on Nature Research policies, see our Editorial Policies and the Editorial Policy Checklist.

\section{Statistics}

For all statistical analyses, confirm that the following items are present in the figure legend, table legend, main text, or Methods section. $\mathrm{n} / \mathrm{a}$ Confirmed

$\bigotimes$ The exact sample size $(n)$ for each experimental group/condition, given as a discrete number and unit of measurement

$\square$ A statement on whether measurements were taken from distinct samples or whether the same sample was measured repeatedly

$\triangle$ The statistical test(s) used AND whether they are one- or two-sided

Xnly common tests should be described solely by name; describe more complex techniques in the Methods section.

$\triangle \square$ A description of all covariates tested

Х $\square$ A description of any assumptions or corrections, such as tests of normality and adjustment for multiple comparisons

$\square$ A full description of the statistical parameters including central tendency (e.g. means) or other basic estimates (e.g. regression coefficient) AND variation (e.g. standard deviation) or associated estimates of uncertainty (e.g. confidence intervals)

$\varnothing$ For null hypothesis testing, the test statistic (e.g. $F, t, r$ ) with confidence intervals, effect sizes, degrees of freedom and $P$ value noted

$\bigotimes \square \begin{aligned} & \text { For null hypothesis testing, the test statistic } \\ & \text { Give } P \text { values as exact values whenever suitable. }\end{aligned}$

Х $\square$ For Bayesian analysis, information on the choice of priors and Markov chain Monte Carlo settings

Х $\square$ For hierarchical and complex designs, identification of the appropriate level for tests and full reporting of outcomes

Х $\square$ Estimates of effect sizes (e.g. Cohen's $d$, Pearson's $r$ ), indicating how they were calculated

\section{Our web collection on statistics for biologists contains articles on many of the points above.}

\section{Software and code}

Policy information about availability of computer code

Data collection Cryo-EM: ThermoFisher EPU, Gatan Latitude S; Unfolding Assay: TECAN safire2 plate reader

Data analysis GraphPad Prism v8.2.1; Cryo-EM: RELION v3.1, CTFFIND v4.1.9; Structure Visualization: Chimera v1.11.2, ChimeraX v0.9, PyMol v2.4.0; Model Building: COOT v0.89, Phenix.refine v1.15.2; MotionCor2 v1.3.2; CTFFIND4 v4.1.10.

For manuscripts utilizing custom algorithms or software that are central to the research but not yet described in published literature, software must be made available to editors and

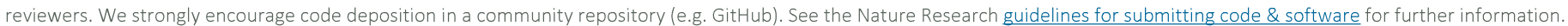

\section{Data}

Policy information about availability of data

All manuscripts must include a data availability statement. This statement should provide the following information, where applicable:

- Accession codes, unique identifiers, or web links for publicly available datasets

- A list of figures that have associated raw data

- A description of any restrictions on data availability

Cryo-EM maps have been deposited in the Electron Microscopy Data Bank (EMDB, www.ebi.ac.uk/pdbe/emdb/) under the accession codes EMDB-23449 (p97-Npl4/ Ufd1-Ubn-Eos-CHAPSO, Class 1), EMDB-23450 (p97-Npl4/Ufd1-Ubn-Eos-CHAPSO, Class 2), EMDB-23451 (p97-Npl4/Ufd1-Ubn-Eos-CHAPSO, Class 3), EMDB-23446 (p97-Npl4/Ufd1-Ubn-Eos-FOM, Class 1), EMDB-23447 (p97-Npl4/Ufd1-Ubn-Eos-FOM, Class 2), EMDB-23448 (p97-Npl4/Ufd1-Ubn-Eos-FOM, Class 3), EMDB-23443 (p97-Npl4/Ufd1-Ub6-FOM, Class 1), EMDB-23444 (p97-Npl4/Ufd1-Ub6-FOM, Class 2), EMDB-23445 (p97-Npl4/Ufd1-Ub6-FOM, Class 3), EMDB-23442 (p97-Npl4/ Ufd1-Ub6-NMS-873-FOM), EMDB-23452 (p97-Npl4/Ufd1-Ubn-Eos-NMS-873-CHAPSO), EMDB-23453 (p97-Npl4/Ufd1-Ubn-Eos-NMS-873(substoichiometric)CHAPSO, Class 1), EMDB-23454 (p97-Npl4/Ufd1-Ubn-Eos-NMS-873(substoichiometric)-CHAPSO, Class 2), EMDB-23455 (p97-Npl4/Ufd1-Ubn-EosNMS-873(substoichiometric)-CHAPSO, Class 3), EMDB-23456 (p97-Npl4/Ufd1-Ubn-Eos-NMS-873(substoichiometric)-CHAPSO, Class 4), EMDB-23457 (p97-Npl4/ 
Ufd1-Ubn-Eos-NMS-873(substoichiometric)-CHAPSO, Class 5), EMDB-23458 (p97-Npl4/Ufd1-Ubn-Eos-NMS-873(substoichiometric)-CHAPSO, Class 6). The atomic models have been deposited in the Protein Data Bank (PDB, www.rcsb.org) under the accession codes 7LN5 (p97-Npl4/Ufd1-Ubn-Eos-CHAPSO, Class 1), 7LN6 (p97Npl4/Ufd1-Ubn-Eos-CHAPSO, Class 2), 7LN2 (p97-Npl4/Ufd1-Ubn-Eos-FOM, Class 1), 7LN3 (p97-Npl4/Ufd1-Ubn-Eos-FOM, Class 2), 7LN4 (p97-Npl4/Ufd1-Ubn-EosFOM, Class 3), 7LMZ (p97-Npl4/Ufd1-Ub6 -FOM, Class 1), 7LNO (p97-Npl4/Ufd1-Ub6-FOM, Class 2), 7LN1 (p97-Npl4/Ufd1-Ub6-FOM, Class 3), 7LMY (p97-Npl4/Ufd1Ub6-NMS-873-FOM). Source data are provided with this paper.

\section{Field-specific reporting}

Please select the one below that is the best fit for your research. If you are not sure, read the appropriate sections before making your selection.

$\bigotimes$ Life sciences Behavioural \& social sciences Ecological, evolutionary \& environmental sciences

For a reference copy of the document with all sections, see nature.com/documents/nr-reporting-summary-flat.pdf

\section{Life sciences study design}

All studies must disclose on these points even when the disclosure is negative.

\begin{tabular}{|c|c|}
\hline Sample size & The work performed here did not involve statistical analyses that would be impacted by sample size. \\
\hline Data exclusions & No data was excluded. \\
\hline Replication & The unfolding assays were performed with three technical replicates. All attempts at replication were successful. \\
\hline Randomization & Randomization is not relevant to this study, because no grouped samples were involved. \\
\hline Blinding & Blinding is not relevant to this study, because no grouped samples were involved. \\
\hline
\end{tabular}

\section{Reporting for specific materials, systems and methods}

We require information from authors about some types of materials, experimental systems and methods used in many studies. Here, indicate whether each material, system or method listed is relevant to your study. If you are not sure if a list item applies to your research, read the appropriate section before selecting a response.

\begin{tabular}{|c|c|}
\hline$n / a$ & Involved in the study \\
\hline$凶$ & $\square$ Antibodies \\
\hline Х & Eukaryotic cell lines \\
\hline 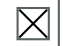 & Palaeontology and archaeology \\
\hline$凶$ & Animals and other organisms \\
\hline Х & Human research participants \\
\hline Х & $\square$ Clinical data \\
\hline$凶$ & $\square$ Dual use research of concern \\
\hline
\end{tabular}

Methods n/a Involved in the study

\ $\square$ chip-seq

\ $\square$ Flow cytometry

$\bigotimes \mid \square$ MRI-based neuroimaging 